\title{
Use of microhabitats affects butterfly assemblages in a rural landscape
}

\author{
Elton Orlandin ${ }^{1,3}$; Mônica Piovesan ${ }^{1,4}$; Fernanda Maurer D’Agostini2 ${ }^{2,5}$ \& Eduardo Carneiro ${ }^{1,6}$ \\ 1 Universidade Federal do Paraná (UFPR), Departamento de Zoologia (DZO0), Laboratório de Estudos de Lepidoptera Neotropical (LABLEPNEO). \\ Curitiba, PR, Brasil. \\ 2 Universidade do Oeste de Santa Catarina (UNOESC), Departamento de Ciências Biológicas e da Saúde. Joaçaba, SC, Brasil. \\ 3 ORCID: http://orcid.org/0000-0002-1987-9727. E-mail: orlandinelton@gmail.com \\ ${ }^{4}$ ORCID: http://orcid.org/0000-0003-3367-9358. E-mail:monica367piovesan@gmail.com \\ ${ }^{5}$ ORCID: http://orcid.org/0000-0002-1115-6153. E-mail: fernanda.dagostini@unoesc.edu.br \\ ${ }^{6}$ ORCID: http://orcid.org/0000-0002-4796-0020. E-mail: eduardo.carneiro@ufpr.br
}

\begin{abstract}
Landscapes composed of small rural properties may support highly heterogeneous habitat, because they often cover distinct types of land uses adjacent to surrounding forest fragments. Many butterfly species may benefit from this kind of landscape, as very distinct microhabitats can be found in a very restricted spatial scale. To better understand how different microhabitats are related to fragmentation in rural landscapes the present study collected the butterfly fauna in 18 sampling point sites, representing distinct types of forest edges and forest interiors. Although closely located, these sites showed no spatial autocorrelation. Instead, a major distinction in species richness and composition was found among forest interior and edge habitats while no significant difference was found in species composition among distinct edge types. Therefore, the high segregation of butterfly assemblages found in a very restricted geographic scale suggests the presence of two different groups of butterflies that respond independently to forest fragmentation, the forest interior assemblages and forest edge assemblages. This distinction of butterfly assemblages related to forest interior and forest edges were already reported, but our results highlights that these differences are found mostly due to species turnover between those habitats. In other words, both microhabitat types present a high number of specialized species compared to a smaller fraction of generalist species that may occurs in both microhabitats. In the case of Atlantic Forest, the species of special conservation concern are those true specialized in forest interior habitats and not those specialized in forest edges, thus the present study corroborates the importance of sampling different microhabitats when studying fragmentation processes, both inside and outside of fragments. Although forest edges may present different kinds of habitat types, species present along border tend to be as heterogeneous as species present in different locations inside the forest. This information should be considered in sampling designs of biodiversity essays that focus on a more consistent representation of local diversity.
\end{abstract}

Key-Words. Atlantic Rainforest; Landscape fragmentation; Host plants; Species list.

\section{INTRODUCTION}

The increase in land exploitation for agricultural use have been identified as one of the main causes of habitat fragmentation (Foley, 2005; Foley et al., 2011). This phenomena increases the isolation and the number of small habitat patches, as well as decreases the original area size of natural habitats (Fahrig, 2003), thus affecting the organisms diversity and distribution (Prugh et al., 2008; Foley et al., 2011; Gibson et al., 2013; Ibáñez et al., 2014; Haddad et al., 2015). All these effects however, are dependent of how different landscape variables change across geographical scales (Brown Jr. \& Hutchings, 1997; Driscoll et al., 2013; Prugh et al., 2008; Verbeylen et al., 2003).
Studies using butterflies as models have demonstrated that local habitat fragmentation can affect their abundance, richness, composition, and diversity (Brown Jr. \& Hutchings, 1997; Bobo et al., 2006; Uehara-Prado et al., 2007; Ribeiro et al., 2008; Uehara-Prado et al., 2009; Bonebrake et al., 2010; Collier et al., 2010; Ribeiro et al., 2012; Robinson et al., 2014; Filgueiras et al., 2016). Most importantly, local butterfly distribution is closely associated with habitat conditions as impacted by habitat fragmentation, such as fragment interior vs. fragment edges (Ribeiro et al., 2012; Brito et al., 2014; Filgueiras et al., 2016). This occurs because species that feed as adults on fruits, decomposing matter or bird excrement, find these resources mainly inside the forest, while nectarivorous species find most of the food resources in the canopy, 
on the edges or in open areas (Brown Jr. \& Hutchings, 1997; Devries \& Walla, 2001; Hill et al., 2001; Brown Jr. \& Freitas, 2002). As distinct types of matrices surrounding fragments may influence the availability of food resources to butterflies, more complex landscapes may offer greater resource diversity (Tews et al., 2004). Therefore, it is expected that rural landscapes under predominance of small farms can harbour more rich and complex assemblages when compared to the extensive monocultural and urban landscapes (Fahrig et al., 2015; Iserhard et al., 2018). This is because small rural properties tend to have different kinds of land occupations, promoting peculiar characteristics, which enable population maintenance and species interaction (Fahrig et al., 2011, 2015).

In addition, the transition area between the fragment and the surrounding matrix may provide differentiated food resources for some groups of insects, relative to those found within the fragment and in the matrix (Landis et al., 2000; Poggio et al., 2010). These areas, the fragment edges, usually have pioneering plant species (Rigueira et al., 2012) and unique micro-climates (Lawson et al., 2014) that form peculiar microhabitats, attracting not only nectarivores, but also predators and other herbivorous insects (Didham et al., 1996; Jokimäki et al., 1998; Albrecht et al., 2010). Fragments surrounded by matrices composed of different occupations (e.g., abandoned areas, crop lands, or roads) present these differentiated transition areas, which form microhabitats at different levels of complexity (e.g., different types of resources). Several butterfly species, for example, are recognized for inhabiting these environments, from where they extract food, both at the larval and adult stages (Brown Jr. \& Hutchings, 1997; Brown Jr. \& Freitas, 2002).

This study aimed to verify whether the butterfly species richness and composition in a rural fragmented landscapes is influenced by these kinds of microhabitat, thus testing the following hypotheses: (1) Despite of their close proximity, the butterfly assemblages have distinct species richness and composition in different microhabitats present in a fragmented landscape; (2) forest edges and interior have distinct butterfly richness and composition, because butterfly species usually prefer for a particular microhabitat type and (3) different edges types presents distinct butterfly richness and composition.

\section{MATERIALS AND METHODS}

\section{Study site}

The study was conducted in the municipality of Joaçaba $\left(27^{\circ} 10^{\prime} 41.0^{\prime \prime} \mathrm{S}, 51^{\circ} 30^{\prime} 17.0^{\prime \prime} \mathrm{W}\right)$, in the western region of Santa Catarina State, southern Brazil (Fig. 1a). This region is broadly characterized by its rural landscape, with small urban areas (Maté et al., 2015). Small farms predominate in the rural landscape, some of them raising livestock such as cattle, pigs, and poultry, while the others grow corn, beans, rice, tobacco, soy, apple, and oranges (Begnini \& Almeida, 2016). The forest fragments are relicts from a transition area (ecotone) between the
Araucaria forest and Deciduous forest (Vibrans et al., 2012). The climate is mesothermal humid with a hot summer (according to Köppen-Geiger climatic classification), the average annual temperature is $18^{\circ} \mathrm{C}$, annual rainfall of about $2,000 \mathrm{~mm}$, relative annual humidity average is $76 \%$ (Alvares et al., 2013), and an altitude of range $700-830 \mathrm{~m}$ (Google Earth, 2016).

\section{Sampling}

Butterfly assemblages were measured in 18 sample sites representing four microhabitat types: forest interiors $(n=6)$; road edges: edge of the fragment closer to the road ( $n=4)$; farmland edges: crops of soybean and corn and cattle ranching $(n=4)$; and abandoned edges: early-regrowth vegetation areas $(n=4)$ (Fig. 2). The focus of the present study was to measure distribution of these butterfly assemblages in a very fine geographical scale. Therefore, these 18 sample sites were choosen in three fragments, being some of them more closely located to each other than to others (Fig. 1b-d, Appendix I). Sample sites in the same fragment were distant from each other by a minimum of $50 \mathrm{~m}$ meters distance when representing distinct microhabitats, or at least $100 \mathrm{~m}$ distance when representing the same microhabitat. Inside the forest, butteflies were captured in a radius up to $10 \mathrm{~m}$, while in forest edges the butterflies were sampled in a transect up to $30 \mathrm{~m}$.

Sampling was conducted with an entomological net between 08:30 AM and 04:00 PM between January 2016 and March 2017, except in April, June, July, and August, totaling 15 replicates for each sample site. Butterfly sampling was performed at each site for 01:15 hours, following a rotation, resulting a total of $337.5 \mathrm{~h} /$ net per site. Therefore, all sites were equally sampled during different times of the day in the same period of the year. Only the butterfly captured and euthanized were considered in the samples. The specimens were identified based on photographs of type series available in Warren et al. (2013) and/or confirmed by specialists. Voucher specimens are deposited in the "Coleção Entomológica Padre Jesus Santiago Moure (DZUP)".

\section{Statistical analyses}

Considering that butterflies can easily move among the sample sites and use more closely located food resources, the samples in this study are potentially subject to spatial autocorrelation. To determine if this was the case, a Mantel test was employed using a Euclidean distance matrix to represent the geographic distances between samples and a similarity matrix based on BrayCurtis index to represent species composition.

Later, we measured the richness and composition of butterfly assemblages at each sample site. The butterfly richness was estimated using the interpolation and extrapolation methodology proposed by Chao \& Jost (2012), available in the iNEXT package (Hsieh et al., 2016). 

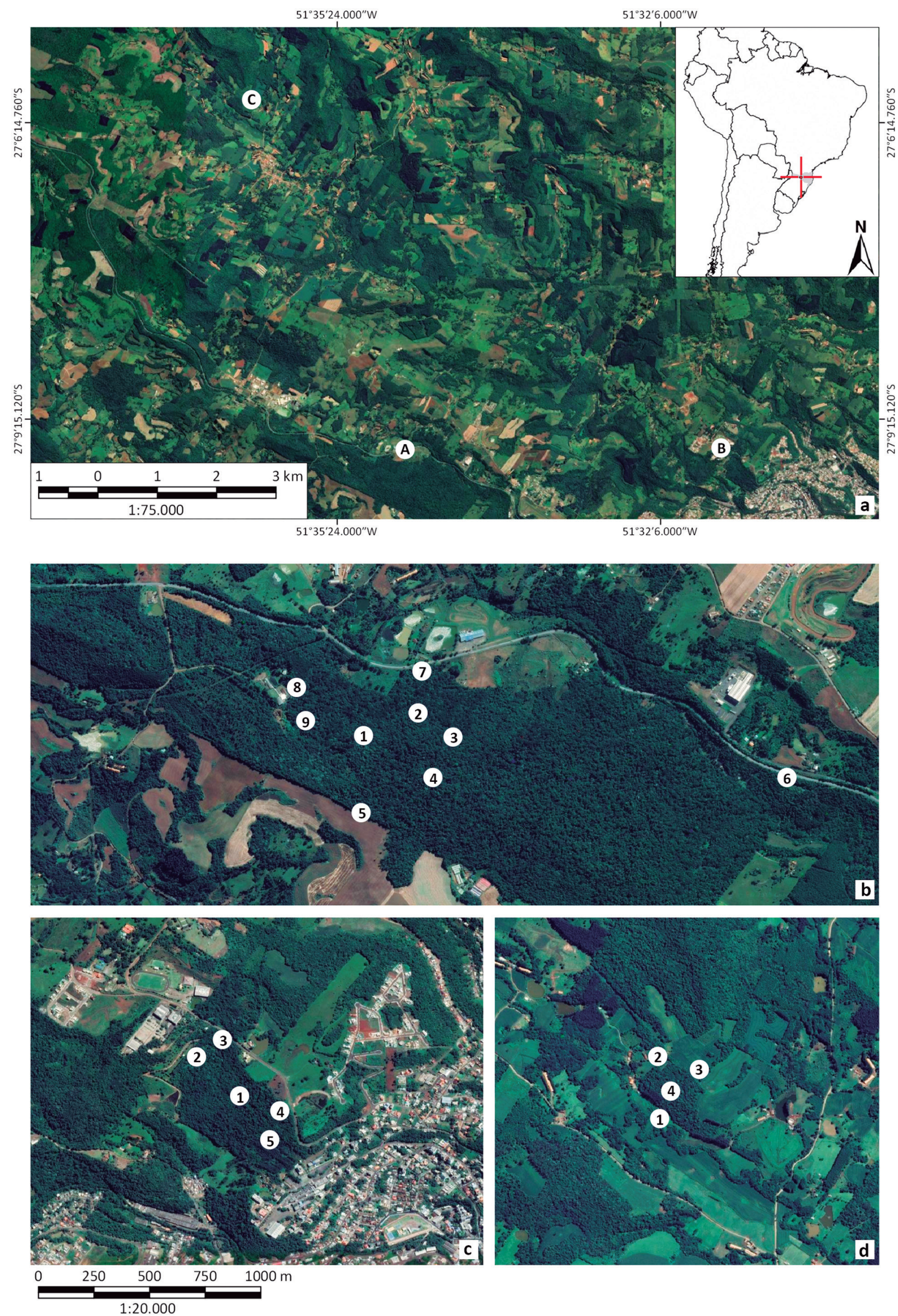

Figure 1. Location of the study area in the Joaçaba Municipality, Santa Catarina State, Brazil. (a) Fragments sampled; (b-c) Distribution of collection points of A, B and C fragments. 

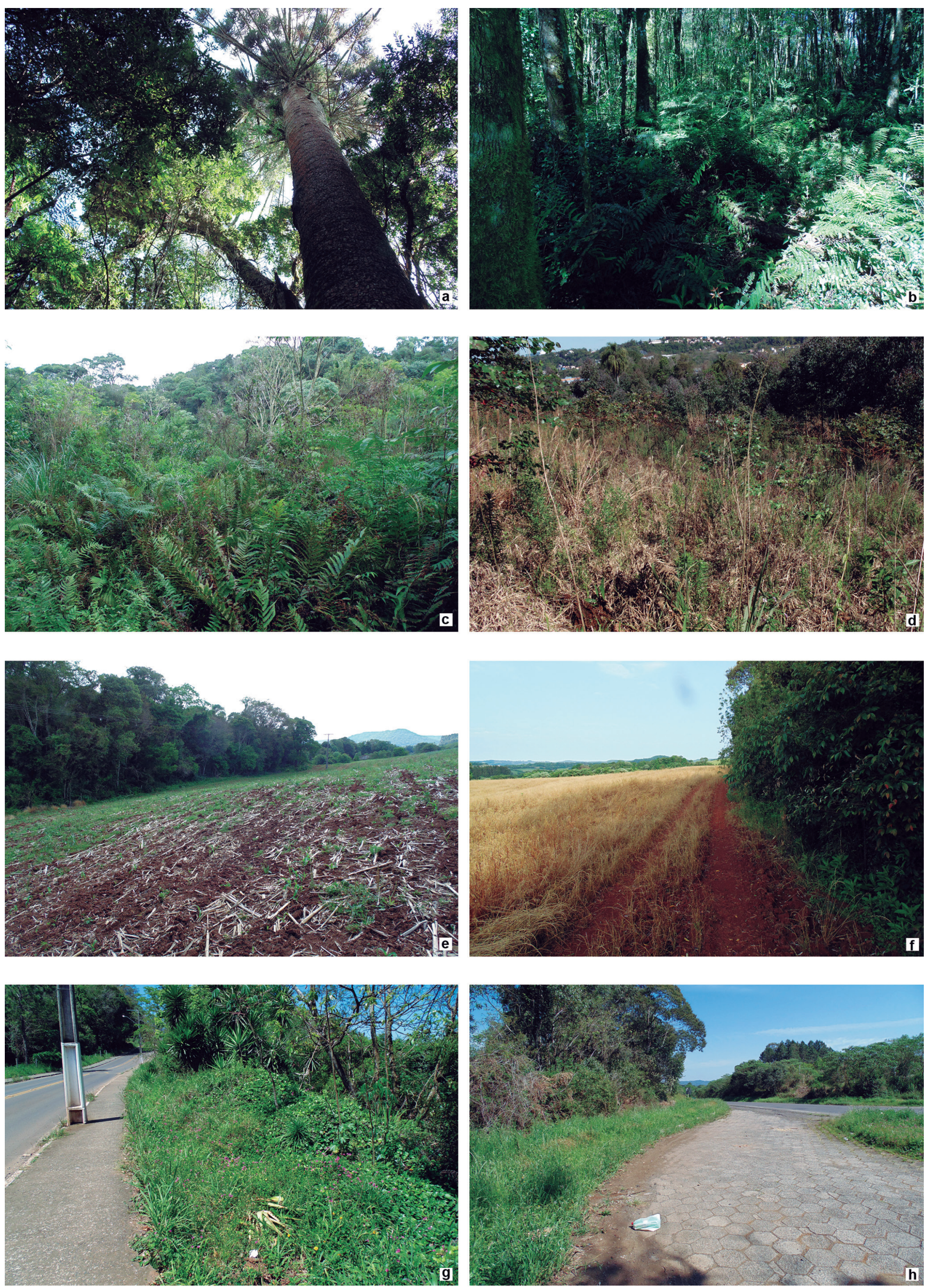

Figure 2. Characterization of the sampled microhabitats in a rural landscape in Southern Brazil. $(a, b)$ forest interior; $(c, d)$ abandoned edge; (e, f) farmland edge; $(g, h)$ road edge. 
This method is particularly efficient to estimates the differences in species richness estimated from communities with distinct structure (e.g., abundances distribution). Non-metric multidimentional scaling (NMDS) based on the Bray-Curtis similarity index was used to access assemblage composition at different microhabitats. This method generates a scatter plot in which closely localized samples (e.g., clustered) exhibit similar fauna composition. Thus, distances between sample sites can be used as surrogates of composition dissimilarity (Melo \& Hepp, 2008). A PERMANOVA test ( $\mathrm{n}=999$ permutations) was performed to test the significance of microhabitat type in shaping the butterfly species composition. The PERMANOVA was performed for two distinct datasets in our study, since the fragment interior showed a very distinct species composition when compared to all other microhabitat types. Therefore, after testing the whole dataset, a subsequent analysis was performed after removing the forest interior samples.

Additionally, we partitioned the Bray-Curtis coefficient into two measurements to test if any of the microhabitat types are distinct in terms of species turnover ( $\beta_{\text {turn }}$-diversity) and nestedness ( $\beta_{\text {nest }}$-diversity) (Baselga, 2013). These results were used to infer whether the differences in species compostion are due to the segregation of different species at different microhabitats (turnover) or because one microhabitat have only a smaller amount of the same species as the other (nestedness). Therefore, if all microhabitats have a large amout of specialists butterflies a higher $\beta_{\text {turn }}$-diversity is expected. On the contrary, when most of the species in a microhabitat are generalists (e.g., found across other microhabitats) a higher $\beta_{\text {nest }}$-diversity is expected. Also to complement this goal, we employed the INDVAL test to verify how many butterfly species present close ecological affinities with any microhabitat type. The INDVAL yields a maximum value when all specimens of a given species are recorded in only one type of habitat and in all samples representing this habitat, despite other species abundances (Dufrene \& Legendre, 1997). Since all forest edges revealed no significant changes in the species composition, we only scored microhabitats as forest edges or forest interior. In this test, we considered only species with $\geq 10$ individuals, thus totalling 190 species tested. The species that presented significant value were compared with the literature's observations regarding adult habits and larvae host plants. All analyses were performed in the $R$ environment ( $R$ Core Team, 2015) using the package vegan (Oksanen et al., 2017), betapart (Baselga, 2013) and labdsv (Roberts, 2016).

\section{RESULTS}

A total of 7,941 butterflies belonging to 431 species were recorded. Twenty-nine species were later recorded during occasional collects totalling 460 species; these species were attached to the species list, but not accounted in the statistical analyses (Appendix II). The Mantel test showed no correlation between species composition and the geographical proximity of sample sites $(R=0.07, p>0.05)$, indicating that sample sites are not spatial autocorrelated.

Comparisons among butterfly richness revealed that different microhabitat types do play a role on butterfly species richness patterns (Fig. 3). Assemblages are richer in forest edges than the forest interiors, with the abandoned edges being the richest, followed by the road edge and the farmland edge (Fig. 4, Appendix III). Also a major distinction in species composition is found among forest interior and edges $\left(R^{2}=0.36476, p<0.001\right.$, Fig. 5a), but in this case the different types of edges revealed no sig-
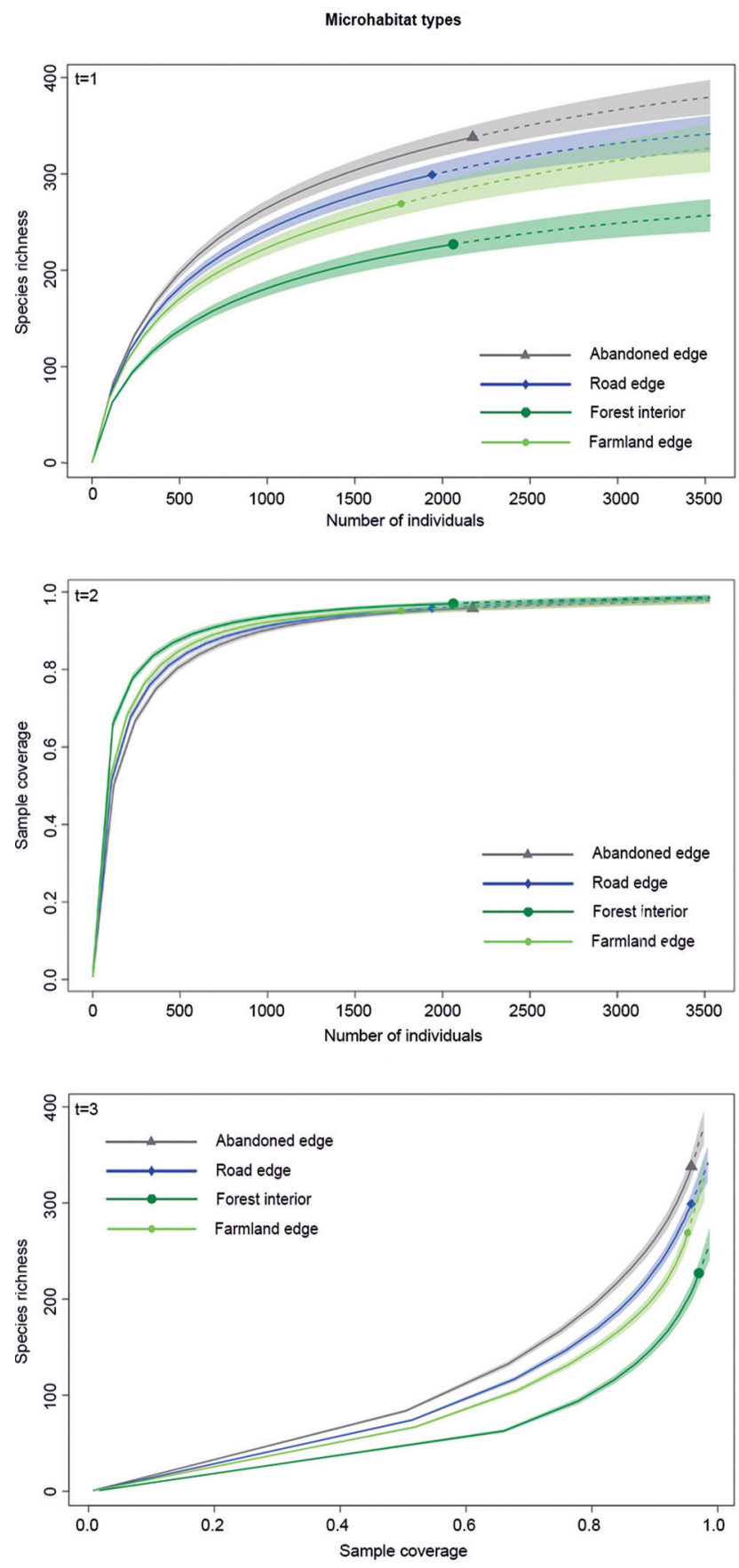

Figure 3. Results of integrated coverage-based rarefaction-extrapolation (Chao \& Jost, 2012) of butterfly richness between microhabitat types in a rural landscape in Southern Brazil (confidence interval $=95 \%$ ). Continuous lines (rarefaction), dotted lines (extrapolation). 


\section{Sampled sites of A, B and C fragments}
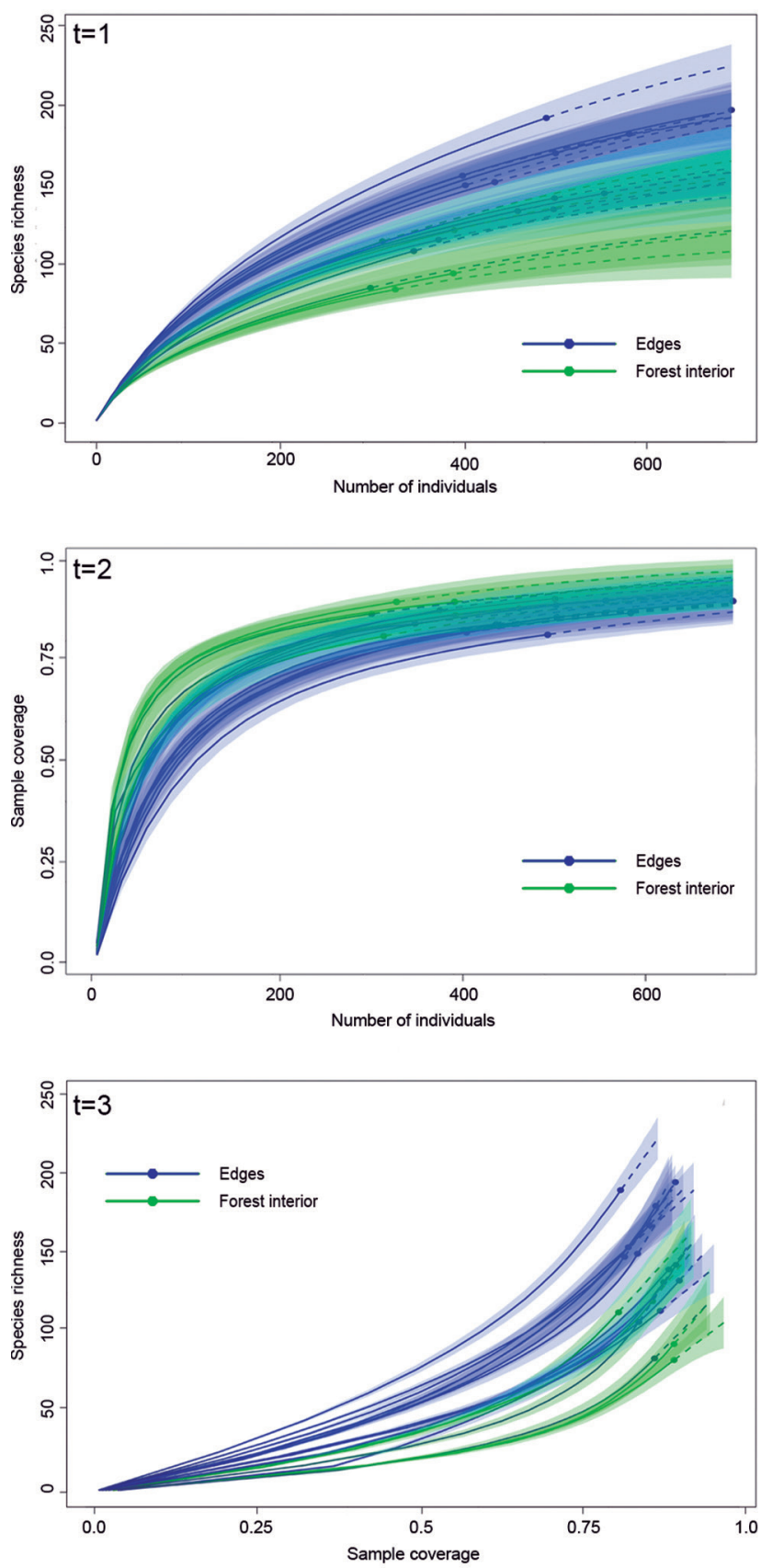

Figure 4. Results of integrated coverage-based rarefaction-extrapolation (Chao \& Jost, 2012) of butterfly richness between 18 sampled points in a rural landscape in Southern Brazil (confidence interval $=95 \%$ ). Continuous lines (rarefaction), dotted lines (extrapolation).

nificant differences between each other, even when the samples from the forest interior were removed from the analyses $\left(R^{2}=0.14894, p=0.909\right.$, Fig. $\left.5 b\right)$. In both types of microhabitats, beta-diversity is mostly represented by species turnover (Forest interior: $\beta_{\text {turn }}=0.5$; Forest edges: $\beta_{\text {turn }}=0.56 ; p=0.58$ ) instead of nestedness (Forest interior: $\beta_{\text {nest }}=0.03$; Forest edges: $\beta_{\text {nest }}=0.04 ; p=0.67$ ), showing that the differences between habitat types is mostly represented by specialists species instead of generalists species. By comparing microhabitat types, the fragment edges show a much larger number of indicator species $(n=68)$, than the forest interior $(n=20)$ (Appendix IV).
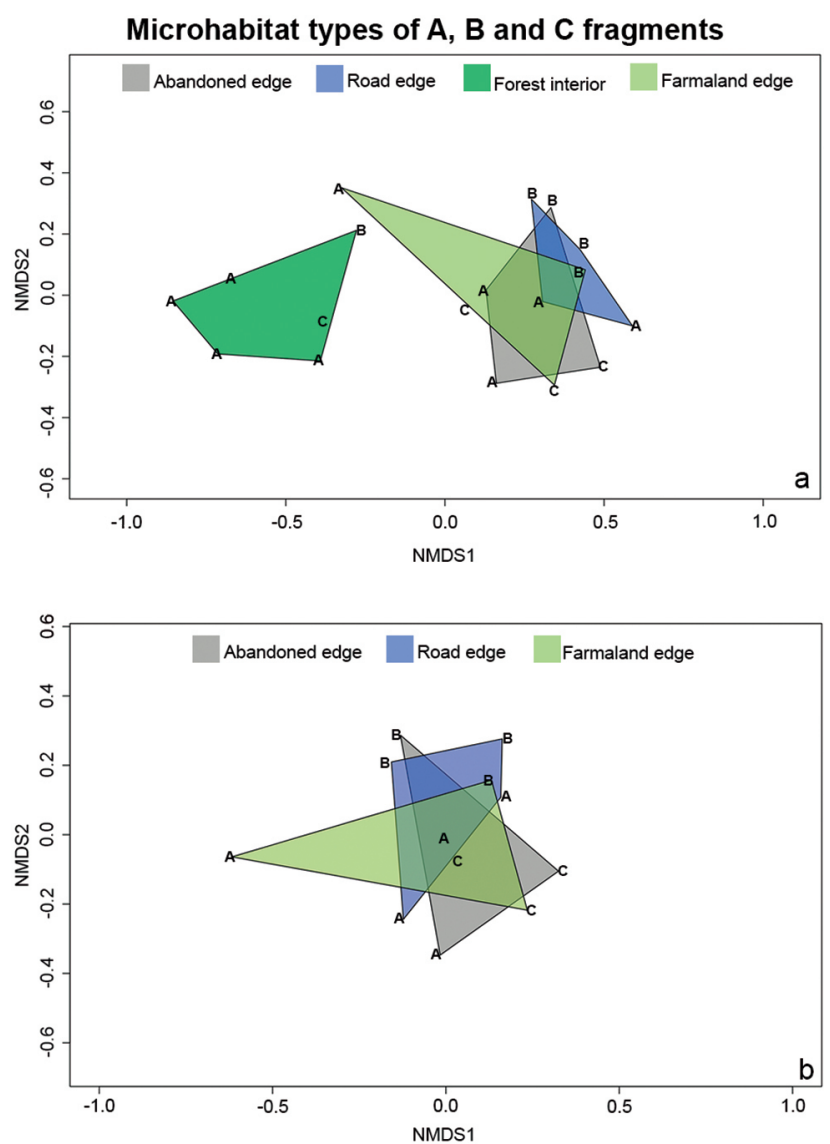

Figure 5. Non-metric multidimensional scaling (NMDS), plots representing butterfly assemblages composition measured in distinct types of microhabitats (abandoned edge, road edge, farmland edge and forest interior) in a rural landscape in Southern Brazil. a) Including forest interior sampled sites (Stress $=0.11) ; b$ ) Excluding forest interior sampled sites (Stress $=0.14$ ). Forest interior butterfly communities are distinct from all edge communities, but all edge habitats support similar butterfly communities despite microhabitat differences (even when interior samples are removed from the data analysis).

\section{DISCUSSION}

The fragmentation impacts of natural environments have been extensively studied in different animal and botanical groups (Uehara-Prado et al., 2007; Buchmann et al., 2013; Sancha et al., 2014; Filgueiras et al., 2016; Justino et al., 2016). In general, several studies already demonstrated how the type of matrix surrounding a fragmented landscape may influence on species richness and composition (Gascon et al., 1999; Steffan-Dewenter, 2003; Vieira et al., 2009; Öckinger et al., 2012; Driscoll et al., 2013). The phenomena associated with these patterns are mostly related to species dispersal, colonization and extinction. However, few studies tested how the use of distinc microhabitat in a fragmented landscapes may influence on the assemblage structure of highly mobile organisms. Considering, for example, that sites inside fragments and sites along fragment border present very distinct habitats traits over a narrow geographical distance, highly mobile organisms such as winged insects could easily make use of both microhabitat types. Our study results showed, using distinct assemblage measurements, that this is not the case of butterfly assemblages. 
Butterflies are widely known to have special preference for specific microhabitats (Brown Jr. \& Hutchings, 1997; Devries \& Walla, 2001; Hill et al., 2001; Brown Jr. \& Freitas, 2002; Uehara-Prado et al., 2007; Ribeiro et al., 2012), such as shady environments (Hill et al., 2001; Brown Jr. \& Freitas, 2002), hilltops (Prieto \& Dahners, 2006; Carneiro et al., 2014); or to fly very close to their host plants (Rutowski, 1991). Most frequently (although not always) this association with microhabitats is based on the presence and abundance of adult and/or larval food resources (Hamer et al., 2006). According to our data, the assemblages sampled within microhabitats are more similar when compared among microhabitats. Therefore, the structure and distribution of butterfly assemblages in a fragment may be very heterogeneous, even when this fragment is deeply reduced in size (Ribeiro et al., 2008).

This pattern cannot be explained by their geographical proximity between sample sites. Actually, even delimiting sample sites in a very narrow distance between each other $(<100 \mathrm{~m})$, we could not find a spatial bias in our assemblages. In general, forest edges concentrated greater species richness than the forest interior, and consequently a higher number of significant indicator species. This difference could occur due to the higher concentration of food resources offered to butterflies at the forest borders. The abundance of flowers attractive to butterflies inside the forest is scarce when compared to the forest edges, where several pioneering plant species bloom mainly from the Asteraceae and Rubiaceae family (Silberbauer-Gottsberger \& Gottsberger, 1988; Andersson et al., 2002; Brown Jr. \& Freitas, 2002; Ramírez, 2004). Such phanerogams are concentrated to a greater or lesser abundance around fragments, depending on which type of edge is found. For example, while abandoned edges are occupied by pioneer vegetation, farmers extend their crop fields closer to the fragment edge, thereby reducing the abundance of pioneer vegetation. Therefore, a greater richness is likely to be found in the abandoned habitat due to the greater abundance and diversity of flowers of this pioneer vegetation richness. Brown \& Hutchings (1997) observed a similar pattern in the Amazon forest fragments, that is, small fragments surrounded by homogeneous areas (burned or pasture) and interiors of large fragments presenting low richness when compared with fragments that contained areas in regrowth and flowers in abundance. Similarly, Öckinger et al. (2012) found that butterfly species' richness is higher in fragments surrounded by matrixes whose vegetation was more similar to the forest fragment. Hence, the quality of forest edges is of great relevance in order to maximize the species richness in fragmented landscapes.

Nevertheless, it is important to highlight that butterfly species richness is not always a good descriptor of habitat quality (Shuey et al., 2017). Instead, species composition has shown to be more sensitive measurement to detect differences between habitat types (UeharaPrado et al., 2007; Truxa \& Fiedler, 2012; Filgueiras et al., 2019). Although we could not find a significant difference in the species composition between different types of edges, (despite of their difference in species richness), the differences between forest interior and edges are remarkable. This distinction of butterfly assemblages related to forest interior and forest edges were already reported, including those of fruit-feeding butterfly (Brown Jr. \& Hutchings, 1997; Uehara-Prado et al., 2007; Ribeiro et al., 2012; Filgueiras et al., 2016). Moreover, our results highlights that these differences are found mostly due to species turnover between those habitats. In other words, both types of microhabitat have a larger fraction of specialized species and only a smaller set of species can be found inhabiting forest interior and forest edges. This pattern is the opposite to those found in the Northern Hemisphere, in which the structure of butterfly assemblages across fine scale habitat use is mostly nested (Summerville et al., 2002; Trivellini et al., 2016). Most likely, the higher turnover rate observed here was produced by the behavior of butterflies. The species commonly found around the fragment hardly perch or forage inside the forest, or when they do, they should use the canopy stratum instead (Hill et al., 2001). The opposite behavior is observed to forest interior species, who usually avoids flying in habitats with high luminosity rates. These species are known to be adapted to shady and humid microclimates, frequently presenting cryptic behaviours and/or coloration (Uehara-Prado \& Freitas, 2009, Iserhard et al., 2018).

Evidently, in the case of Atlantic Forest the species of special conservation concern are those specialized in forest interior habitats and not those specialized in forest edges (Brown Jr. \& Hutchings, 1997; Ribeiro et al., 2012; Filgueiras et al., 2016). However, not all of them are reliable indicators of habitat quality. The abundance of some Ithomiini species for example, respond only to the presence of small pockets of humidity generated inside the fragments, instead of habitat quality (Brown Jr. \& Freitas, 2002). The same could occur with some Satyrinae species whose larvae feed on grasses that invades the understory of strongly modified fragments. On the contrary, species such as Celaenorrhinus eligius punctiger (Burmeister, 1878) may indicate habitat quality because both larvae and adults feed on typical understory food resources (De Jong, 1982; Brown, 1992). Although our results pointed to a relevant number of interior forest indicators, we believe it is possible that several forest specialists are no longer present in the region due to their sensitiveness to disturbances (Hill et al., 2001; Cleary \& Genner, 2004, Filgueiras et al., 2019). This hypothesis would also explain the lower number of species found in forest interiors when compared to forest edges.

The present study corroborates the importance of sampling different microhabitats when studying fragmentation processes, both inside and outside of fragments. Although forest edges may present different kinds of habitat types, species present along border tend to be as heterogeneous as species present in different locations inside the forest. This information should be considered in sampling designs of biodiversity essays that focus on a more consistent representation of local diversity. 


\section{ACKNOWLEDGEMENTS}

We would like to thank Diego Rodrigo Dolibaina, Fernando Maia Silva Dias, Marlon Paluch, Mirna Martins Casagrande, Olaf Hermann Hendrik Mielke, Thamara Zacca Bispo Taumaturgo and Wildio Ikaro da Graça Santos for help with species identification.

\section{REFERENCES}

Albrecht, M.; Schmid, B.;Obrist, M.K.; Schüpbach, B.;Kleijn, D.\& Duelli, P. 2010. Effects of ecological compensation meadows on arthropod diversity in adjacent intensively managed grassland. Biological Conservation, 143(3): 642-649.

Alvares, C.A.; Stape, J.L.; Sentelhas, P.C.; de Moraes Gonçalves, J.L. \& Sparovek, G. 2013. Köppen's climate classification map for Brazil. Meteorologische Zeitschrift, 22(6): 711-728.

Andersson, S.; Nilsson, L.A.; Groth, I. \& Bergström, G. 2002. Floral scents in butterfly-pollinated plants: Possible convergence in chemical composition. Botanical Journal of the Linnean Society, 140(2): 129-153.

Baselga, A. 2013. Separating the two components of abundance-based dissimilarity: Balanced changes in abundance vs. abundance gradients. Methods in Ecology and Evolution, 4(6): 552-557.

Beccaloni, G.W.; Viloria, A.L. Hall, S.K. \& Robinson, G.S. 2008. Catalogue of the hostplants of the Neotropical butterflies / Catálogo de las plantas huésped de las mariposas neotropicales. Zaragoza, Sociedad Entomológica Aragonesa. 536p.

Begnini, S. \& Almeida, L.E.D.F. de. 2016. Grau de desenvolvimento regional dos municípios da mesorregião oeste catarinense: caracterização e classificação. Interações, Campo Grande, 17(4): 547-560.

Biezanko, C.M. \& Mielke, 0.H.H. 1973. Contribuição ao estudo faunístico dos Hesperiidae americanos. IV. Espécies do Rio Grande do Sul, Brasil, com notas taxonômicas e descrições de espécies novas (Lepidoptera). Acta Biológica Paranaense, 2: 51-102.

Biezanko, C.M.; Ruffinelli, A. \& Link, D. 1974. Plantas y otras sustancias alimenticias de las orugas de los lepidópteros uruguayos. Revista do Centro de Ciências Rurais, 4: 107-148.

Bobo, K.S.; Waltert, M.; Fermon, H.; Njokagbor, J. \& Mühlenberg, M. 2006. From forest to farmland: butterfly diversity and habitat associations along a gradient of forest conversion in Southwestern Cameroon. Journal of Insect Conservation, 10(1): 29-42.

Bonebrake, T.C.; Ponisio, L.C.; Boggs, C.L. \& Ehrlich, P.R. 2010. More than just indicators: A review of tropical butterfly ecology and conservation. Biological Conservation, 143(8): 1831-1841.

Brito, M.M.; Ribeiro, D.B.; Raniero, M.; Hasui, É.; Ramos, F.N. \& Arab, A. 2014. Functional composition and phenology of fruit-feeding butterflies in a fragmented landscape: Variation of seasonality between habitat specialists. Journal of Insect Conservation, 18(4): 547-560.

Brown Jr., K.S. \& Freitas, A.V.L. 2002. Butterfly communities of urban forest fragments in Campinas, São Paulo, Brazil: Structure, instability, environmental correlates, and conservation. Journal of Insect Conservation, 6(4): 217-231.

Brown Jr., K.S. \& Hutchings, R.W. 1997. Disturbance, fragmentation, and the dynamics of diversity in Amazonian forest butterflies. In: Laurance, W.F. \& Bierregaard Jr., R.0. Tropical forest remnants: Ecology, management, and conservation of fragmented communities. Chicago, Chicago University Press. p. 91-110.

Brown, K. 1992. Borboletas da Serra do Japi: Diversidade, habitats, recursos alimentares e variação temporal. In: Morellto, L.P.C. História Natural da
Serra do Japi: Ecologia e Preservação de uma área Florestal no Sudeste do Brasil. Campinas, UNICAMP. p. 142-186.

Buchmann, C.M.; Schurr, F.M.; Nathan, R. \& Jeltsch, F. 2013. Habitat loss and fragmentation affecting mammal and bird communities. The role of interspecific competition and individual space use. Ecological Informatics, 14: $90-98$

Carneiro, E.; Mielke, 0.H.H.; Casagrande, M.M. \& Fiedler, K. 2014. Skipper richness (Hesperiidae) along elevational gradients in Brazilian Atlantic Forest. Neotropical Entomology, 43(1): 27-38.

Chao, A. \& Jost, L. 2012. Coverage-based rarefaction and extrapolation: Standardizing samples by completeness rather than size. Ecology, 93(12): 2533-2547.

Cleary, D.F.R. \& Genner, M.J. 2004. Changes in rain forest butterfly diversity following major ENSO-induced fires in Borneo. Global Ecology and Biogeography, 13(2): 129-140.

Collier, N.; Gardner, M.; Adams, M.; McMahon, C.R.; Benkendorff, K. \& Mackay, D.A. 2010. Contemporary habitat loss reduces genetic diversity in an ecologically specialized butterfly: Contemporary habitat loss and genetic diversity. Journal of Biogeography, 37(7): 1277-1287.

De Jong, R. 1982. Secondary sexual characters in Celaenorrhinus and the delimitation of the genus (Lepidoptera, Hesperiidae). Journal of Natural History, 16(5): 695-705.

Devries, P.J. \& Walla, T.R. 2001. Species diversity and community structure in neotropical fruit-feeding butterflies. Biological Journal of the Linnean Society, 74(1): 1-15.

Didham, R.K.; Ghazoul, J.; Stork, N.E. \& Davis, A.J. 1996. Insects in fragmented forests: A functional approach. Trends in Ecology \& Evolution, 11(6): 255-260.

Driscoll, D.A.; Banks, S.C.; Barton, P.S.; Lindenmayer, D.B. \& Smith, A.L. 2013. Conceptual domain of the matrix in fragmented landscapes. Trends in Ecology \& Evolution, 28(10): 605-613.

Duarte, M.; Robbins, R.K. \& Mielke, 0. H. H. 2005. Immature stages of Calycopis caulonia (Hewitson, 1877) (Lepidoptera, Lycaenidae, Theclinae, Eumaeini), with notes on rearing detritivorous hairstreaks on artificial diet. Zootaxa, 1063: 1-31

Dufrene, M. \& Legendre, P. 1997. Species Assemblages and Indicator Species: The Need for a Flexible Asymmetrical Approach. Ecological Monographs, 67(3): 345.

Fahrig, L. 2003. Effects of habitat fragmentation on biodiversity. Annual Review of Ecology, Evolution, and Systematics, 487-515.

Fahrig, L.; Baudry, J.; Brotons, L.; Burel, F.G.; Crist, T.0.; Fuller, R.J.; Sirami, C.; Siriwardena, G.M. \& Martin, J.-L. 2011. Functional landscape heterogeneity and animal biodiversity in agricultural landscapes: Heterogeneity and biodiversity. Ecology Letters, 14(2): 101-112.

Fahrig, L.; Girard, J.; Duro, D.; Pasher, J.; Smith, A.; Javorek, S.; King, D.; Lindsay, K.F.; Mitchell, S. \& Tischendorf, L. 2015. Farmlands with smaller crop fields have higher within-field biodiversity. Agriculture, Ecosystems \& Environment, 200: 219-234.

Filgueiras, B.K.C.; Melo, D.H.A.; Leal, I.R.; Tabarelli, M.; Freitas, A.V.L. \& Iannuzzi, L. 2016. Fruit-feeding butterflies in edge-dominated habitats: Community structure, species persistence and cascade effect. Journal of Insect Conservation, 20(3): 539-548.

Filgueiras, B.K.C.; Melo, D.H.A.; Uehara-Prado, M.; Freitas, A.V.L.; Leal, I.R. \& Tabarelli, M. 2019. Compensatory dynamics on the community structure of fruit-feeding butterflies across hyper-fragmented Atlantic forest habitats. Ecological Indicators, 98: 276-284.

Foley, J.A. 2005. Global Consequences of Land Use. Science, 309(5734): 570-574.

Foley, J.A.; Ramankutty, N.; Brauman, K.A.; Cassidy, E.S.; Gerber, J.S.; Johnston, M.; Mueller, N.D.; O'Connell, C.; Ray, D.K.; West, P.C.; Balzer, C.; Bennett, 
E.M.; Carpenter, S.R.; Hill, J.; Monfreda, C.; Polasky, S.; Rockström, J.; Sheehan, J.; Siebert, S.; Tilman, D. \& Zaks, D.P.M. 2011. Solutions for a cultivated planet. Nature, 478(7369): 337-342.

Freitas, A.V.; Kaminski, L.A.; Mielke, O.H.H.; Barbosa, E.P. \& Silva-Brandao, K.L. 2012. A new species of Yphthimoides (Lepidoptera: Nymphalidae: Satyrinae) from the southern Atlantic forest region. Zootaxa, 3526: 31-44.

Gascon, C.; Lovejoy, T.E.; Bierregaard Jr., R.O.; Malcolm, J.R.; Stouffer, P.C.; Vasconcelos, H.L.; Laurance, W.F; Zimmermann, B.; Tocher, M. \& Borges, S. 1999. Matrix habitat and species richness in tropical forest remnants. Biological Conservation, 91(2-3): 223-229.

Gibson, L.; Lynam, A.J.; Bradshaw, C.J.A.; He, F.; Bickford, D.P.; Woodruff, D.S.; Bumrungsri, S. \& Laurance, W.F. 2013. Near-Complete Extinction of Native Small Mammal Fauna 25 Years After Forest Fragmentation. Science, 341(6153): 1508-1510.

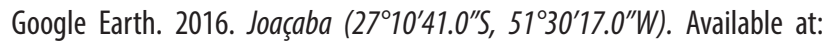
https://www.google.com.br/intl/pt-BR/earth. Access in: 28/10/2017.

Haddad, N.M.; Brudvig, L.A.; Clobert, J.; Davies, K.F.; Gonzalez, A.; Holt, R.D.; Lovejoy, T.E.; Sexton, J.0.; Austin, M.P.; Collins, C.D.; Cook, W.M.; Damschen, E.I.; Ewers, R.M.; Foster, B.L.; Jenkins, C.N.; King. A.J.; Faurance, W.F.; Levey, D.J.; Margules, C.R.; Melbourne, B.A.; Nicholls, A.0.; Orrock, J.L.; Song, D.-X. \& Townshend, J.R. 2015. Habitat fragmentation and its lasting impact on Earth's ecosystems. Science Advances, 1(2): e1500052.

Hamer, K.C.; Hill, J.K.; Benedick, S.; Mustaffa, N.; Chey, V.K. \& Maryati, M. 2006. Diversity and ecology of carrion- and fruit-feeding butterflies in Bornean rain forest. Journal of Tropical Ecology, 22(01): 25-33.

Hill, J.; Hamer, K.; Tangah, J. \& Dawood, M. 2001. Ecology of tropical butterflies in rainforest gaps. Oecologia, 128(2): 294-302.

Hsieh, T.C.; Ma, K.H. \& Chao, A. 2016. iNEXT: An R package for rarefaction and extrapolation of species diversity (Hill numbers). Methods in Ecology and Evolution, 7(12): 1451-1456.

Ibáñez, I.; Katz, D.S.W.; Peltier, D.; Wolf, S.M. \& Connor Barrie, B.T. 2014. Assessing the integrated effects of landscape fragmentation on plants and plant communities: The challenge of multiprocess-multiresponse dynamics. Journal of Ecology, 102(4): 882-895.

Iserhard, C.A.; Duarte, L.; Seraphim, N. \& Freitas, A.V.L. 2018. How urbanization affects multiple dimensions of biodiversity in tropical butterfly assemblages. Biodiversity and Conservation, 28(3): 621-638.

Jokimäki, J.; Huhta, E.; Itämies, J. \& Rahko, P. 1998. Distribution of arthropods in relation to forest patch size, edge, and stand characteristics. Canadian Journal of Forest Research, 28(7): 1068-1072.

Justino, C.E.L.; dos Santos, E.F. \& Noll, F.B. 2016. Diversity of Tiphiidae (Insecta: Hymenoptera) in the fragmented Brazilian semi-deciduous Atlantic Forest. Journal of Insect Conservation, 20(3): 417-431.

Landis, D.A.; Wratten, S.D. \& Gurr, G.M. 2000. Habitat management to conserve natural enemies of arthropod pests in agriculture. Annual Review of Entomology, 45(1): 175-201.

Lawson, C.R.; Bennie, J.; Hodgson, J.A.; Thomas, C.D. \& Wilson, R.J. 2014. Topographic microclimates drive microhabitat associations at the range margin of a butterfly. Ecography, 37(8): 732-740.

Maté, C.; Micheleti, T. \& Santiago, A.G. 2015. Cidades de pequeno porte em Santa Catarina: Uma reflexão sobre planejamento territorial. Revista Políticas Públicas \& Cidades, 3(2): 28-47.

Melo, A.S. \& Hepp, L.U. 2008. Ferramentas estatísticas para análises de dados provenientes de biomonitoramento. Oecologia brasiliensis, 12(3): 463-486.

Öckinger, E.; Lindborg, R.; Sjödin, N.E. \& Bommarco, R. 2012. Landscape matrix modifies richness of plants and insects in grassland fragments. Ecography, 35(3): 259-267.
Oksanen, J.; Blanchet, F.G.; Friendly, M.; Kindt, R.; Legendre, P.; McGlinn, D.; Minchin, P.R.; O'Hara, R.B.; Simpson, G.L.; Solymos, P.; Stevens, M.H.H.; Szoecs, E.; Wagner, H. 2017. Vegan: community ecology package, $R$ package version 2.4-3. https://CRAN.R-project.org/package=vegan.

Poggio, S.L.; Chaneton, E.J. \& Ghersa, C.M. 2010. Landscape complexity differentially affects alpha, beta, and gamma diversities of plants occurring in fencerows and crop fields. Biological Conservation, 143(11): 2477-2486.

Prieto, C. \& Dahners, H.D. 2006. Eumaeini (Lepidoptera: Lycaenidae) del cerro San Antonio: Dinámica de la riqueza y comportamiento de "Hilltopping". Revista Colombiana de Entomología, 32(2): 179-190.

Prugh, L.R.; Hodges, K.E.; Sinclair, A.R.E. \& Brashares, J.S. 2008. Effect of habitat area and isolation on fragmented animal populations. Proceedings of the National Academy of Sciences, 105(52): 20770-20775.

R Core Team. 2015. R: A language and environment for statistical computing. Vienna, R Foundation for Statistical Computing. Available at: http:// www.R-project.org. Access in: 27/08/2017.

Ramírez, N. 2004. Ecology of pollination in a tropical Venezuelan savanna. Plant Ecology, 173(2): 171-189.

Ribeiro, D.B.; Batista, R.; Prado, P.I.; Brown, K.S. \& Freitas, A.V.L. 2012. The importance of small scales to the fruit-feeding butterfly assemblages in a fragmented landscape. Biodiversity and Conservation, 21(3): 811-827.

Ribeiro, D.B.; Prado, P.l.; Brown Jr., K.S. \& Freitas, A.V.L. 2008. Additive partitioning of butterfly diversity in a fragmented landscape: Importance of scale and implications for conservation. Diversity and Distributions, 14(6): 961-968.

Rigueira, D.M.G.; Molinari, A.L.M.; Mariano, D.L.S.; Reis, R.M.; Portugal, A.B.; Santana, N.S. \& Santos, R.A. 2012. Influência da distância da borda e do adensamento foliar sobre a abundância de plantas pioneiras em um fragmento de floresta tropical submontana na Estação Ecológica de Wenceslau Guimarães (Bahia, Brasil). Acta Botanica Brasilica, 26(1): 197-202.

Roberts, D.W. 2016. Package 'labdsv'. Available at: https://cran.r-project.org/ src/contrib/Archive/labdsv. Access in: 28/09/2017.

Robinson, N.; Kadlec, T.; Bowers, M.D. \& Guralnick, R.P. 2014. Integrating species traits and habitat characteristics into models of butterfly diversity in a fragmented ecosystem. Ecological Modelling, 281: 15-25.

Rutowski, R.L. 1991. The evolution of male mate-locating behavior in butterflies. The American Naturalist, 138(5): 1121-1139.

Sancha, N.U. de la, Higgins, C.L.; Presley, S.J. \& Strauss, R.E. 2014. Metacommunity structure in a highly fragmented forest: Has deforestation in the Atlantic Forest altered historic biogeographic patterns? Diversity and Distributions, 20(9): 1058-1070.

Shuey, J.A.; Labus, P.; Carneiro, E.V.; Dias, F.M.S.; Leite, L.A.R. \& Mielke, 0.H.H. 2017. Butterfly communities respond to structural changes in forest restorations and regeneration in lowland Atlantic Forest, Paraná, Brazil. Journal of Insect Conservation, 21(3): 545-557.

Silberbauer-Gottsberger, I. \& Gottsberger, G. 1988. A polinização de plantas do cerrado. Revista Brasileira de Biologia, 48(4): 651-663.

Steffan-Dewenter, I. 2003. Importance of habitat area and landscape context for species richness of bees and wasps in fragmented orchard meadows. Conservation Biology, 17(4): 1036-1044.

Summerville, K.S.; Veech, J.A. \& Crist, T.0. 2002. Does variation in patch use among butterfly species contribute to nestedness at fine spatial scales? Oikos, 97(2): 195-204.

Tews, J.; Brose, U.; Grimm, V.; Tielbörger, K.; Wichmann, M.C.; Schwager, M. \& Jeltsch, F. 2004. Animal species diversity driven by habitat heterogeneity/diversity: The importance of keystone structures: Animal species diversity driven by habitat heterogeneity. Journal of Biogeography, 31(1): 79-92. 
Trivellini, G.; Polidori, C.; Pasquaretta, C.; Orsenigo, S. \& Bogliani, G. 2016. Nestedness of habitat specialists within habitat generalists in a butterfly assemblage. Insect Conservation and Diversity, 9(6): 495-505.

Truxa, C. \& Fiedler, K. 2012. Down in the flood? How moth communities are shaped in temperate floodplain forests: Floodplain forest moths. Insect Conservation and Diversity, 5(5): 389-397.

Uehara-Prado, M. \& Freitas, A.V.L. 2009. The effect of rainforest fragmentation on species diversity and mimicry ring composition of ithomiine butterflies. Insect Conservation and Diversity, 2(1): 23-28.

Uehara-Prado, M.; Brown, K.S. \& Freitas, A.V.L. 2007. Species richness, composition and abundance of fruit-feeding butterflies in the Brazilian Atlantic Forest: Comparison between a fragmented and a continuous landscape. Global Ecology and Biogeography, 16(1): 43-54.

Uehara-Prado, M.; Fernandes, J. de 0.; Bello, A. de M.; Machado, G.; Santos, A.J.; Vaz-de-Mello, F.Z. \& Freitas, A.V.L. 2009. Selecting terrestrial arthropods as indicators of small-scale disturbance: A first approach in the Brazilian Atlantic Forest. Biological Conservation, 142(6): 1220-1228.
Verbeylen, G.; De Bruyn, L.; Adriaensen, F. \& Matthysen, E. 2003. Does matrix resistance influence Red squirrel (Sciurus vulgaris L. 1758) distribution in an urban landscape? Landscape Ecology, 18(8): 791-805.

Vibrans, A.C.; Sevegnani, L.; Gasper, A.L. de \& Lingner, D.V. 2012. Inventário florístico florestal de Santa Catarina. Vol. 2: Floresta estacional decidual. Blumenau, Universidade Regional de Blumenau.

Vieira, M.V.; Olifiers, N.; Delciellos, A.C.; Antunes, V.Z.; Bernardo, L.R.; Grelle, C.E.V. \& Cerqueira, R. 2009. Land use vs. Fragment size and isolation as determinants of small mammal composition and richness in Atlantic Forest remnants. Biological Conservation, 142(6): 1191-1200.

Warren, A.D.; Davis, K.J.; Grishin, N.V.; Pelham, J.P. \& Stangeland, E.M. 2013. Butterflies of America. Illustrated Lists of American Butterflies. http:// butterfliesofamerica.com/intro.htm.

Zacca, T.; Paluch, M.; Siewert, R.; Freitas, A.; Barbosa, E.; Mielke, 0.H.H. \& Casagrande, M.M. 2017. Revision of Godartiana Forster (Lepidoptera: Nymphalidae), with the description of a new species from northeastern Brazil. Austral Entomology, 56: 169-190. 


\section{APPENDIX I}

Geographic coordinates of sampled sites in the studied area.

$F=$ Fragments $(A, B, C) ; T=$ Microhabitat types $(I=$ forest interior, $F=$ farmland edge, $R=$ road edge, $A b=$ abandoned edge)

\begin{tabular}{|c|c|c|c|c|c|}
\hline$F / T$ & Latitude & Longitude & $F / T$ & Latitude & Longitude \\
\hline A1/I & -27.163431 & -51.584744 & B1/I & -27.162906 & -51.521150 \\
\hline A2/I & -27.162514 & -51.582222 & $B 2 / F$ & -27.160906 & -51.523441 \\
\hline A3/I & -27.163406 & -51.580575 & $\mathrm{~B} 3 / \mathrm{R}$ & -27.160169 & -51.521833 \\
\hline A4/I & -27.165284 & -51.581486 & $B 4 / R$ & -27.163364 & -51.519058 \\
\hline $\mathrm{A} 5 / \mathrm{F}$ & -27.166906 & -51.584844 & B5/Ab & -27.165044 & -51.520150 \\
\hline A6/R & -27.16569 & -51.565428 & C1/Ab & -27.101853 & -51.607444 \\
\hline$A 7 / R$ & -27.160314 & -51.582244 & $\mathrm{C} 2 / \mathrm{F}$ & -27.099936 & -51.607708 \\
\hline$A 8 / A b$ & -27.161467 & -51.587906 & $\mathrm{C} 3 / \mathrm{F}$ & -27.100242 & -51.606400 \\
\hline$A 9 / A b$ & -27.163155 & -51.587621 & $C 4 / I$ & -27.100831 & -51.607111 \\
\hline
\end{tabular}




\section{APPENDIX II}

\section{List of butterflies (Papilionoidea) sampled in the sites studied, Joaçaba, Santa Catarina, Brazil. Fragments (A, B, C). * Indicates species sampled by chance}

\begin{tabular}{|c|c|c|c|c|c|c|c|c|c|c|c|c|c|c|c|c|c|c|c|}
\hline FAMILY/Subfamily/Tribe/Specie & A1 & A2 & A3 & A4 & A5 & A6 & A7 & A8 & A9 & B1 & B2 & B3 & B4 & B5 & C1 & $\mathrm{C} 2$ & C3 & C4 & Total \\
\hline \multicolumn{20}{|l|}{ HESPERIIDAE } \\
\hline \multicolumn{20}{|l|}{ Eudaminae } \\
\hline \multicolumn{20}{|l|}{ Eudamini } \\
\hline 1 Aguna asander asander (Hewitson, 1867) & 0 & 0 & 0 & 0 & 0 & 1 & 0 & 0 & 0 & 0 & 0 & 0 & 0 & 0 & 0 & 1 & 0 & 0 & 2 \\
\hline $2{ }^{*}$ Aguna glaphyrus (Mabille, 1888) & - & - & - & - & - & - & - & - & - & - & - & - & - & - & - & - & - & - & - \\
\hline 3 Astraptes aulus (Plötz, 1881) & 1 & 0 & 0 & 0 & 0 & 0 & 0 & 0 & 1 & 0 & 0 & 0 & 0 & 0 & 0 & 1 & 0 & 0 & 3 \\
\hline 4 Astraptes enotrus (Stoll, [1781]) & 0 & 0 & 0 & 0 & 0 & 0 & 0 & 0 & 1 & 0 & 0 & 0 & 0 & 0 & 0 & 1 & 0 & 0 & 2 \\
\hline 5 Astraptes erycina (Plötz, 1881) & 0 & 0 & 0 & 1 & 0 & 0 & 0 & 0 & 2 & 0 & 0 & 0 & 0 & 0 & 1 & 0 & 0 & 0 & 4 \\
\hline 6 Cecropterus dorantes dorantes (Stoll, [1790] & 0 & 0 & 2 & 0 & 0 & 1 & 3 & 2 & 0 & 0 & 1 & 1 & 2 & 0 & 1 & 0 & 3 & 1 & 17 \\
\hline 7 Cecropterus doryssus albicuspis (Herrich-Schäffer, 1869) & 0 & 0 & 0 & 0 & 2 & 2 & 0 & 0 & 0 & 1 & 0 & 0 & 0 & 0 & 0 & 0 & 0 & 0 & 5 \\
\hline 8 Cecropterus rica (Evans, 1952) & 0 & 0 & 0 & 0 & 0 & 0 & 0 & 1 & 0 & 1 & 0 & 0 & 0 & 0 & 0 & 0 & 0 & 0 & 2 \\
\hline 9 Cecropterus zarex (Hübner, 1818) & 1 & 0 & 0 & 0 & 1 & 0 & 0 & 0 & 5 & 0 & 0 & 0 & 0 & 0 & 1 & 0 & 1 & 0 & 9 \\
\hline 10 Oechydrus evelinda (Butler, 1870) & 1 & 0 & 0 & 0 & 0 & 0 & 1 & 0 & 0 & 0 & 0 & 0 & 0 & 0 & 1 & 0 & 0 & 0 & 3 \\
\hline 11 Polygonus leo leo (Gmelin, [1790]) & 0 & 0 & 0 & 0 & 0 & 0 & 0 & 0 & 0 & 0 & 0 & 0 & 0 & 0 & 1 & 0 & 0 & 0 & 1 \\
\hline 12 Polygonus savigny savigny (Latreille, [1824]) & 1 & 0 & 0 & 0 & 0 & 0 & 1 & 1 & 1 & 0 & 0 & 1 & 0 & 0 & 1 & 0 & 0 & 1 & 7 \\
\hline 13 Proteides mercurius mercurius (Fabricius, 1787) & 0 & 0 & 0 & 0 & 0 & 1 & 0 & 0 & 0 & 0 & 0 & 0 & 0 & 0 & 0 & 0 & 0 & 0 & 1 \\
\hline 14 Spicauda procne (Plötz, 1880) & 0 & 1 & 1 & 0 & 0 & 1 & 4 & 1 & 1 & 0 & 5 & 2 & 3 & 2 & 2 & 1 & 0 & 1 & 25 \\
\hline 15 Spicauda simplicius (Stoll, [1790]) & 1 & 0 & 0 & 0 & 0 & 2 & 2 & 1 & 0 & 0 & 1 & 1 & 7 & 0 & 5 & 3 & 0 & 0 & 23 \\
\hline 16 Spicauda teleus (Hübner, 1821) & 2 & 0 & 0 & 0 & 2 & 2 & 10 & 4 & 5 & 0 & 7 & 4 & 4 & 9 & 9 & 3 & 6 & 0 & 67 \\
\hline 17 Spicauda zagorus (Plötz, 1880) & 0 & 0 & 0 & 0 & 0 & 1 & 0 & 0 & 0 & 0 & 0 & 0 & 0 & 0 & 0 & 0 & 0 & 0 & 1 \\
\hline $18{ }^{*}$ Telegonus alardus alardus (Stoll, 1790) & - & - & - & - & - & - & - & - & - & - & - & - & - & - & - & - & - & - & - \\
\hline 19 Telegonus cretatus adoba (Evans, 1952) & 0 & 1 & 0 & 0 & 0 & 1 & 0 & 0 & 1 & 0 & 0 & 0 & 0 & 0 & 1 & 1 & 0 & 0 & 5 \\
\hline 20 Telegonus creteus siges (Mabille, 1903) & 1 & 0 & 0 & 0 & 0 & 0 & 0 & 0 & 0 & 0 & 0 & 1 & 0 & 0 & 0 & 1 & 0 & 1 & 4 \\
\hline 21 Telegonus elorus (Hewitson, 1867) & 2 & 1 & 1 & 0 & 0 & 3 & 0 & 0 & 0 & 0 & 0 & 0 & 1 & 0 & 0 & 0 & 1 & 0 & 9 \\
\hline 22 Telegonus fulgerator fulgerator (Walch, 1775) & 5 & 0 & 1 & 2 & 1 & 1 & 0 & 2 & 1 & 1 & 0 & 0 & 0 & 0 & 0 & 1 & 2 & 3 & 20 \\
\hline 23 Urbanus esta Evans, 1952 & 0 & 0 & 0 & 0 & 0 & 0 & 0 & 0 & 0 & 1 & 0 & 0 & 1 & 1 & 0 & 0 & 2 & 0 & 5 \\
\hline 24 Urbanus pronta Evans, 1952 & 0 & 0 & 0 & 0 & 0 & 0 & 0 & 0 & 2 & 0 & 0 & 0 & 1 & 0 & 0 & 0 & 0 & 0 & 3 \\
\hline 25 Urbanus proteus (Linnaeus, 1758) & 0 & 0 & 0 & 0 & 0 & 0 & 0 & 0 & 0 & 0 & 0 & 0 & 1 & 0 & 0 & 0 & 0 & 0 & 1 \\
\hline \multicolumn{20}{|l|}{ Phocidini } \\
\hline 26 Nascus phocus (Cramer, [1777]) & 1 & 0 & 0 & 0 & 0 & 0 & 0 & 0 & 0 & 0 & 0 & 0 & 0 & 0 & 0 & 0 & 0 & 0 & 1 \\
\hline 27 Phocides charon (C. \& R. Felder, 1859) & 0 & 0 & 0 & 0 & 0 & 0 & 0 & 0 & 0 & 0 & 0 & 0 & 0 & 0 & 0 & 0 & 1 & 0 & 1 \\
\hline 28 *Phocides pialia pialia (Hewitson, 1857) & - & - & - & - & - & - & - & - & - & - & - & - & - & - & - & - & - & - & - \\
\hline \multicolumn{20}{|l|}{ Heteropterinae } \\
\hline 29 Dardarina aspila Mielke, 1966 & 0 & 0 & 0 & 0 & 0 & 0 & 1 & 2 & 4 & 0 & 0 & 0 & 0 & 0 & 6 & 0 & 1 & 0 & 14 \\
\hline 30 Dardarina rana Evans, 1955 & 0 & 0 & 0 & 0 & 0 & 0 & 0 & 0 & 0 & 0 & 0 & 0 & 0 & 0 & 2 & 2 & 0 & 0 & 4 \\
\hline \multicolumn{20}{|l|}{ Hesperiinae } \\
\hline \multicolumn{20}{|l|}{ Hesperiini } \\
\hline 31 Anthoptus epictetus (Fabricius, 1793) & 6 & 0 & 4 & 0 & 1 & 1 & 32 & 16 & 29 & 1 & 5 & 10 & 2 & 1 & 12 & 15 & 2 & 2 & 139 \\
\hline 32 Arita arita (Schaus, 1902) & 0 & 1 & 2 & 1 & 2 & 1 & 1 & 1 & 0 & 1 & 0 & 0 & 1 & 0 & 0 & 0 & 0 & 0 & 11 \\
\hline 33 Arita mubevensis (Bell, 1932) & 0 & 0 & 1 & 1 & 0 & 0 & 0 & 0 & 0 & 0 & 0 & 0 & 0 & 0 & 0 & 0 & 0 & 0 & 2 \\
\hline 34 Artines satyr Evans, 1955 & 0 & 0 & 0 & 0 & 0 & 0 & 0 & 0 & 0 & 0 & 0 & 0 & 0 & 3 & 0 & 0 & 0 & 0 & 3 \\
\hline 35 Callimormus interpunctata (Plötz, 1884) & 22 & 12 & 13 & 1 & 9 & 0 & 5 & 9 & 13 & 13 & 1 & 7 & 0 & 1 & 4 & 2 & 18 & 10 & 140 \\
\hline 36 Callimormus rivera (Plötz, 1882) & 0 & 0 & 0 & 0 & 0 & 6 & 11 & 10 & 7 & 0 & 15 & 8 & 3 & 4 & 11 & 9 & 7 & 0 & 91 \\
\hline $37{ }^{*}$ Calpodes ethlius (Stoll, [1782]) & - & - & - & - & - & - & - & - & - & - & - & - & - & - & - & - & - & - & - \\
\hline 38 Chalcone briquenydan australis Mielke, 1980 & 0 & 0 & 0 & 0 & 0 & 1 & 0 & 0 & 0 & 0 & 0 & 0 & 0 & 0 & 0 & 0 & 0 & 0 & 1 \\
\hline 39 Cobalopsis hazarma (Hewitson, 1877) & 0 & 0 & 0 & 0 & 1 & 2 & 0 & 1 & 1 & 1 & 2 & 0 & 2 & 0 & 2 & 0 & 3 & 0 & 15 \\
\hline 40 Cobalopsis miaba (Schaus, 1902) & 6 & 5 & 6 & 3 & 6 & 0 & 0 & 3 & 1 & 3 & 0 & 0 & 1 & 0 & 0 & 0 & 2 & 5 & 41 \\
\hline 41 Cobalopsis nero (Herrich-Schäffer, 1869) & 0 & 0 & 0 & 0 & 0 & 2 & 0 & 4 & 0 & 3 & 0 & 4 & 7 & 6 & 1 & 0 & 1 & 1 & 29 \\
\hline 42 Cobalopsis vorgia (Schaus, 1902) & 0 & 2 & 2 & 0 & 4 & 0 & 2 & 2 & 2 & 2 & 0 & 1 & 2 & 0 & 0 & 0 & 3 & 1 & 23 \\
\hline 43 Conga chydaea (Butler, 1877) & 3 & 0 & 0 & 0 & 1 & 7 & 2 & 2 & 3 & 4 & 2 & 1 & 0 & 2 & 1 & 3 & 4 & 0 & 35 \\
\hline 44 Conga iheringii (Mabille, 1891) & 0 & 0 & 0 & 0 & 0 & 4 & 4 & 2 & 1 & 0 & 4 & 3 & 0 & 1 & 9 & 1 & 0 & 0 & 29 \\
\hline 45 Conga immaculata (Bell, 1930) & 4 & 1 & 1 & 2 & 3 & 5 & 1 & 3 & 12 & 0 & 2 & 3 & 1 & 2 & 10 & 1 & 4 & 4 & 59 \\
\hline 46 Conga urqua (Schaus, 1902) & 0 & 0 & 0 & 0 & 0 & 0 & 0 & 0 & 1 & 0 & 0 & 0 & 0 & 0 & 0 & 0 & 0 & 0 & 1 \\
\hline 47 Corticea corticea (Plötz, 1883) & 1 & 0 & 0 & 0 & 0 & 0 & 7 & 1 & 1 & 0 & 1 & 1 & 0 & 1 & 2 & 0 & 0 & 0 & 15 \\
\hline 48 Corticea lysias potex Evans, 1955 & 2 & 0 & 0 & 1 & 0 & 10 & 3 & 14 & 24 & 2 & 14 & 31 & 8 & 7 & 19 & 7 & 6 & 1 & 149 \\
\hline
\end{tabular}




\begin{tabular}{|c|c|c|c|c|c|c|c|c|c|c|c|c|c|c|c|c|c|c|c|}
\hline FAMILY/Subfamily/Tribe/Specie & A1 & A2 & A3 & A4 & A5 & A6 & A7 & A8 & A9 & B1 & B2 & B3 & B4 & B5 & C1 & $\mathrm{C} 2$ & C3 & C4 & Total \\
\hline 49 Corticea mendica ssp. n. & 0 & 0 & 2 & 0 & 5 & 0 & 2 & 2 & 4 & 0 & 2 & 0 & 1 & 2 & 3 & 2 & 0 & 0 & 25 \\
\hline 50 Corticea noctis (Plötz, 1883) & 0 & 0 & 1 & 1 & 2 & 0 & 2 & 1 & 3 & 1 & 0 & 2 & 1 & 2 & 0 & 0 & 0 & 0 & 16 \\
\hline 51 Corticea oblinita (Mabille, 1891) & 1 & 0 & 1 & 1 & 0 & 10 & 8 & 6 & 9 & 0 & 4 & 10 & 4 & 5 & 7 & 4 & 1 & 1 & 72 \\
\hline 52 Corticea obscura Mielke, 1969 & 0 & 0 & 0 & 0 & 0 & 0 & 1 & 1 & 0 & 0 & 2 & 3 & 0 & 0 & 0 & 0 & 1 & 0 & 8 \\
\hline 53 Corticea sp. n. & 0 & 0 & 0 & 0 & 4 & 0 & 0 & 0 & 1 & 0 & 1 & 0 & 0 & 0 & 5 & 0 & 0 & 0 & 11 \\
\hline 54 Cumbre meridionalis (Hayward, 1934) & 0 & 0 & 0 & 0 & 0 & 2 & 0 & 1 & 1 & 2 & 2 & 1 & 2 & 0 & 2 & 0 & 1 & 1 & 15 \\
\hline 55 Cymaenes distigma (Plötz, 1882) & 1 & 1 & 0 & 0 & 0 & 0 & 0 & 1 & 0 & 0 & 0 & 0 & 0 & 0 & 0 & 0 & 0 & 0 & 3 \\
\hline 56 Cymaenes gisca Evans, 1955 & 0 & 0 & 0 & 0 & 0 & 1 & 2 & 2 & 0 & 0 & 4 & 0 & 12 & 2 & 1 & 0 & 0 & 0 & 24 \\
\hline 57 Cymaenes laureolus loxa Evans, 1955 & 0 & 0 & 0 & 0 & 0 & 2 & 0 & 0 & 1 & 0 & 0 & 0 & 0 & 0 & 4 & 1 & 1 & 0 & 9 \\
\hline 58 Cymaenes lepta (Hayward, 1939) & 0 & 0 & 0 & 0 & 0 & 0 & 0 & 2 & 0 & 0 & 0 & 1 & 0 & 0 & 1 & 0 & 0 & 1 & 5 \\
\hline 59 Cymaenes odilia odilia (Burmeister, 1878) & 0 & 0 & 0 & 0 & 0 & 0 & 1 & 0 & 0 & 0 & 0 & 0 & 0 & 0 & 0 & 1 & 0 & 0 & 2 \\
\hline 60 Cymaenes perloides (Plötz, 1882) & 0 & 0 & 1 & 1 & 0 & 0 & 1 & 0 & 0 & 0 & 0 & 0 & 0 & 0 & 0 & 0 & 0 & 1 & 4 \\
\hline 61 Cymaenes tripunctata tripunctata (Latreille, [1824]) & 1 & 0 & 0 & 0 & 2 & 4 & 25 & 7 & 8 & 0 & 6 & 4 & 2 & 1 & 10 & 5 & 8 & 0 & 83 \\
\hline 62 Cyclosma altama (Schaus, 1902) & 0 & 0 & 0 & 0 & 0 & 0 & 0 & 0 & 4 & 0 & 0 & 0 & 0 & 0 & 0 & 0 & 0 & 0 & 4 \\
\hline 63 Cynea sp. & 0 & 0 & 0 & 0 & 1 & 0 & 0 & 0 & 0 & 0 & 0 & 0 & 0 & 0 & 0 & 0 & 0 & 0 & 1 \\
\hline 64 *Decinea decinea decinea (Hewitson, 1876) & - & - & - & - & - & - & - & - & - & - & - & - & - & - & - & - & - & - & - \\
\hline 65 Decinea lucifer (Hübner, [1831]) & 0 & 0 & 0 & 0 & 0 & 1 & 1 & 0 & 0 & 0 & 0 & 2 & 1 & 2 & 0 & 0 & 0 & 0 & 7 \\
\hline 66 Dion meda (Hewitson, 1877) & 0 & 0 & 1 & 0 & 0 & 0 & 0 & 0 & 1 & 0 & 0 & 0 & 0 & 0 & 0 & 0 & 0 & 0 & 2 \\
\hline 67 Enosis schausi Mielke \& Casagrande, 2002 & 2 & 0 & 1 & 0 & 4 & 0 & 3 & 0 & 1 & 0 & 0 & 0 & 0 & 0 & 0 & 0 & 0 & 0 & 11 \\
\hline 68 Euphyes leptosema (Mabille, 1891) & 0 & 0 & 0 & 0 & 0 & 0 & 0 & 0 & 0 & 0 & 0 & 0 & 0 & 0 & 2 & 0 & 0 & 0 & 2 \\
\hline 69 Eutychide physcella (Hewitson, [1866]) & 3 & 1 & 2 & 2 & 0 & 1 & 3 & 2 & 7 & 0 & 0 & 0 & 0 & 1 & 0 & 2 & 0 & 0 & 24 \\
\hline 70 *Evansiella cordela (Plötz, 1882) & - & - & - & - & - & - & - & - & - & - & - & - & - & - & - & - & - & - & - \\
\hline 71 Gallio carasta (Schaus, 1902) & 1 & 0 & 1 & 1 & 0 & 0 & 0 & 0 & 0 & 0 & 0 & 0 & 1 & 1 & 0 & 0 & 0 & 0 & 5 \\
\hline 72 Ginungagapus ranesus (Schaus, 1902) & 20 & 1 & 0 & 0 & 3 & 19 & 31 & 4 & 13 & 0 & 12 & 5 & 3 & 13 & 1 & 0 & 2 & 1 & 128 \\
\hline 73 Hansa devergens hydra Evans, 1955 & 1 & 0 & 0 & 0 & 0 & 1 & 0 & 0 & 0 & 0 & 0 & 0 & 2 & 0 & 0 & 0 & 0 & 0 & 4 \\
\hline 74 Hansa hyboma (Plötz, 1886) & 0 & 0 & 0 & 0 & 0 & 0 & 0 & 0 & 0 & 0 & 0 & 0 & 0 & 0 & 0 & 1 & 0 & 0 & 1 \\
\hline 75 Hylephila phyleus phyleus (Drury, [1773]) & 0 & 0 & 0 & 0 & 0 & 1 & 0 & 0 & 0 & 0 & 0 & 4 & 3 & 1 & 6 & 0 & 0 & 0 & 15 \\
\hline 76 Justinia kora (Hewitson, 1877) & 1 & 0 & 0 & 0 & 2 & 1 & 3 & 1 & 5 & 1 & 0 & 3 & 0 & 0 & 1 & 0 & 0 & 1 & 19 \\
\hline 77 Lamponia lamponia (Hewitson, 1876) & 0 & 0 & 0 & 0 & 1 & 1 & 0 & 0 & 0 & 0 & 0 & 0 & 2 & 0 & 0 & 0 & 0 & 0 & 4 \\
\hline 78 Lerema duroca lenta Evans, 1955 & 0 & 0 & 0 & 0 & 0 & 0 & 0 & 1 & 1 & 0 & 0 & 0 & 0 & 0 & 0 & 0 & 0 & 0 & 2 \\
\hline 79 Levina levina (Plötz, 1884) & 1 & 2 & 3 & 1 & 6 & 0 & 1 & 2 & 1 & 0 & 0 & 0 & 5 & 0 & 0 & 0 & 1 & 0 & 23 \\
\hline 80 Libra anatolica (Plötz, 1883) & 0 & 0 & 0 & 0 & 0 & 2 & 0 & 0 & 0 & 0 & 0 & 0 & 0 & 1 & 0 & 0 & 0 & 0 & 3 \\
\hline 81 Lucida lucia lucia (Capronnier, 1874) & 5 & 6 & 3 & 6 & 5 & 0 & 0 & 2 & 2 & 1 & 0 & 2 & 1 & 4 & 0 & 0 & 4 & 5 & 46 \\
\hline 82 Miltomiges cinnamomea (Herrich-Schäffer, 1869) & 0 & 1 & 1 & 0 & 0 & 0 & 0 & 0 & 0 & 0 & 0 & 1 & 0 & 1 & 0 & 0 & 0 & 0 & 4 \\
\hline 83 Mnasitheus chrysophrys (Mabille, 1891) & 0 & 0 & 1 & 0 & 0 & 0 & 0 & 0 & 0 & 1 & 0 & 0 & 1 & 0 & 0 & 0 & 0 & 0 & 3 \\
\hline 84 Mnasitheus gemignanii (Hayward, 1940) & 0 & 0 & 0 & 1 & 0 & 0 & 0 & 0 & 0 & 0 & 0 & 0 & 0 & 0 & 0 & 0 & 0 & 0 & 1 \\
\hline 85 Mnasitheus ritans (Schaus, 1902) & 1 & 3 & 1 & 1 & 3 & 0 & 10 & 8 & 5 & 1 & 0 & 1 & 0 & 1 & 0 & 0 & 1 & 0 & 36 \\
\hline 86 Mnasitheus submetallesces (Hayward, 1940) & 1 & 0 & 0 & 0 & 0 & 4 & 0 & 0 & 0 & 0 & 0 & 0 & 0 & 0 & 0 & 0 & 0 & 0 & 5 \\
\hline 87 Mnasilus allubita (Butler, 1877) & 0 & 0 & 0 & 0 & 0 & 0 & 2 & 0 & 0 & 0 & 0 & 0 & 0 & 0 & 0 & 1 & 1 & 1 & 5 \\
\hline 88 Moeris seth Carneiro, Mielke \& Casagrande, 2015 & 0 & 0 & 0 & 0 & 0 & 1 & 0 & 0 & 0 & 0 & 0 & 0 & 1 & 0 & 0 & 0 & 0 & 0 & 2 \\
\hline 89 Moeris striga striga (Geyer, [1832]) & 0 & 0 & 0 & 0 & 0 & 1 & 0 & 0 & 0 & 0 & 0 & 3 & 0 & 1 & 0 & 0 & 1 & 0 & 6 \\
\hline 90 Morys geisa (Möschler, 1879) & 0 & 0 & 0 & 0 & 0 & 0 & 0 & 0 & 0 & 0 & 0 & 0 & 0 & 1 & 0 & 0 & 0 & 0 & 1 \\
\hline 91 Monca branca Evans, 1955 & 1 & 0 & 0 & 0 & 0 & 1 & 3 & 2 & 0 & 1 & 2 & 6 & 0 & 1 & 8 & 5 & 1 & 1 & 32 \\
\hline 92 *Nastra dryas (Hayward, 1940) & - & - & - & - & - & - & - & - & - & - & - & - & - & - & - & - & - & - & - \\
\hline 93 Nastra lurida (Herrich-Schäffer, 1869) & 0 & 1 & 0 & 0 & 1 & 0 & 1 & 1 & 1 & 0 & 0 & 1 & 0 & 0 & 3 & 5 & 4 & 0 & 18 \\
\hline 94 Neoxeniades scipio scipio (Fabricius, 1793) & 0 & 0 & 0 & 0 & 0 & 0 & 0 & 0 & 0 & 0 & 0 & 0 & 1 & 0 & 0 & 0 & 0 & 0 & 1 \\
\hline 95 Niconiades caeso (Mabille, 1891) & 0 & 0 & 0 & 0 & 0 & 0 & 0 & 1 & 0 & 0 & 0 & 0 & 0 & 0 & 0 & 0 & 0 & 0 & 1 \\
\hline 96 Niconiades merenda (Mabille, 1878) & 3 & 0 & 0 & 2 & 2 & 0 & 0 & 2 & 2 & 0 & 0 & 0 & 0 & 0 & 0 & 0 & 1 & 0 & 12 \\
\hline 97 Nyctelius nyctelius nyctelius (Latreille, [1824]) & 0 & 0 & 0 & 0 & 0 & 0 & 0 & 0 & 0 & 0 & 0 & 0 & 1 & 0 & 0 & 0 & 0 & 0 & 1 \\
\hline 98 Nyctelius paranensis (Schaus, 1902) & 0 & 0 & 0 & 0 & 0 & 1 & 0 & 2 & 2 & 0 & 0 & 0 & 0 & 0 & 0 & 0 & 0 & 0 & 5 \\
\hline 99 Orthos orthos hyalinus (Bell, 1930) & 0 & 0 & 0 & 0 & 1 & 8 & 1 & 0 & 5 & 1 & 1 & 0 & 2 & 0 & 0 & 0 & 0 & 0 & 19 \\
\hline 100 Panoquina ocola ocola (Edwards, 1863) & 0 & 0 & 0 & 0 & 0 & 0 & 0 & 0 & 0 & 0 & 0 & 0 & 0 & 1 & 0 & 0 & 0 & 0 & 1 \\
\hline 101 Papias phainis Godman, [1900] & 0 & 0 & 0 & 0 & 0 & 0 & 0 & 0 & 0 & 0 & 0 & 0 & 0 & 0 & 2 & 0 & 0 & 0 & 2 \\
\hline 102 Paracarystus evansi Hayward, 1938 & 1 & 0 & 0 & 1 & 0 & 0 & 0 & 0 & 1 & 0 & 0 & 0 & 0 & 0 & 0 & 0 & 0 & 0 & 3 \\
\hline 103 Parphorus pseudecorus (Hayward, 1934) & 0 & 0 & 0 & 0 & 0 & 0 & 0 & 0 & 0 & 0 & 0 & 0 & 0 & 0 & 1 & 0 & 0 & 1 & 2 \\
\hline 104 Pheraeus argynnis (Plötz, 1884) & 0 & 0 & 0 & 0 & 0 & 0 & 0 & 0 & 0 & 0 & 0 & 0 & 0 & 0 & 0 & 0 & 1 & 0 & 1 \\
\hline 105 Polites vibex catilina (Plötz, 1886) & 0 & 0 & 0 & 0 & 0 & 5 & 2 & 1 & 0 & 0 & 7 & 4 & 0 & 5 & 4 & 0 & 1 & 0 & 29 \\
\hline 106 Pompeius amblyspila (Mabille, 1898) & 0 & 0 & 0 & 0 & 0 & 1 & 0 & 0 & 0 & 0 & 0 & 0 & 0 & 0 & 0 & 0 & 0 & 0 & 1 \\
\hline 107 Pompeius pompeius (Latreille, [1824]) & 0 & 0 & 0 & 0 & 0 & 2 & 3 & 1 & 0 & 0 & 1 & 6 & 1 & 0 & 3 & 2 & 3 & 0 & 22 \\
\hline 108 Psoralis stacara (Schaus, 1902) & 0 & 0 & 1 & 0 & 5 & 1 & 0 & 0 & 3 & 1 & 0 & 1 & 1 & 0 & 0 & 0 & 0 & 0 & 13 \\
\hline
\end{tabular}


FAMILY/Subfamily/Tribe/Specie

109 Quinta cannae (Herrich-Schäffer, 1869)

110 Remella remus (Fabricius, 1798)

111 Rufocumbre celioi Dolibaina, Mielke \& Casagrande, 2017

112 *Saliana antoninus (Latreille, [1824])

113 Saniba sabina (Plötz, 1882)

114 Saturnus reticulata conspicuus (Bell, 1941)

115 Sodalia coler (Schaus, 1902)

116 Styriodes sp.

117 *Synale hylaspes (Stoll, 1781)

118 Synapte malitiosa antistia (Plötz, 1882)

119 Synapte silius (Latreille, [1824])

120 Thargella caura occulta (Schaus, 1902)

121 Thargella evansi Biezanko \& Mielke, 1973

122 Thespieus ethemides (Burmeister, 1878)

123 Thespieus jora Evans, 1955

124 Thespieus lutetia (Hewitson, [1866])

125 Thracides cleanthes cleanthes (Latreille, [1824])

126 Tigasis fusca (Hayward, 1940)

127 Tirynthia conflua (Herrich-Schäffer, 1869)

128 Tisias lesueur lesueur (Latreille, [1824])

129 Turesis complanula (Herrich-Schäffer, 1869)

130 Vehilius clavicula (Plötz, 1884)

131 Vehilius inca (Scudder, 1872)

132 Vehilius stictomenes stictomenes (Butler, 1877)

133 Vettius artona (Hewitson, 1868)

134 Vettius umbrata (Erschoff, 1876)

135 Vinius letis (Plötz, 1883)

136 Virga austrinus (Hayward, 1934)

137 Virga riparia Mielke, 1969

138 Wallengrenia premnas (Wallengren, 1860)

139 Xeniades orchamus orchamus (Cramer, [1777])

140 Zariaspes mys (Hübner, [1808])

141 Zenis jebus jebus (Plötz, 1882)

142 *Zenis minos (Latreille, [1824])

Pericharini

143 Lycas argentea (Hewitson, [1866])

144 Lychnuchoides ozias ozias (Hewitson, 1878)

145 Orses itea (Swainson, 1821)

146 *Perichares adela (Hewitson, 1867)

147 Perichares aurina Evans, 1955

148 *Perichares lotus (A. Butler, 1870)

149 Perichares seneca seneca (Latreille, [1824])

\section{Pyrginae}

\section{Achlyodini}

150 Achlyodes busirus rioja Evans, 1953

151 Achlyodes mithridates thraso (Hübner, [1807])

152 Aethilla echina coracina Butler, 1870

153 Milanion leucaspis (Mabille, 1878)

154 Pythonides lancea (Hewitson, 1868)

155 Quadrus u-lucida mimus (Mabille \& Boullet, 1917)

156 Zera hyacinthinus servius (Plötz, 1884)

157 Zera tetrastigma erisichthon (Plötz, 1884)

Carcharodini

158 Nisoniades bipuncta (Schaus, 1902)

159 Noctuana diurna (Butler, 1870)

160 Pellicia vecina vecina Schaus, 1902

161 Polyctor polyctor polyctor (Prittwitz, 1868)

162 Staphylus coecatus (Mabille, 1891)

163 Staphylus minor minor Schaus, 1902

164 Staphylus musculus (Burmeister, 1875) $\begin{array}{lllllllllllllllllll}\text { A1 } & \text { A2 } & \text { A3 } & \text { A4 } & \text { A5 } & \text { A6 } & \text { A7 } & \text { A8 } & \text { A9 } & \text { B1 } & \text { B2 } & \text { B3 } & \text { B4 } & \text { B5 } & \text { C1 } & \text { C2 } & \text { C3 } & \text { C4 } & \text { Total }\end{array}$

$\begin{array}{lllllllllllllllllll}0 & 0 & 0 & 0 & 1 & 0 & 0 & 0 & 0 & 0 & 0 & 3 & 0 & 0 & 0 & 0 & 0 & 0 & 4\end{array}$

$\begin{array}{lllllllllllllllllll}2 & 3 & 0 & 2 & 3 & 1 & 0 & 2 & 5 & 0 & 1 & 4 & 5 & 4 & 2 & 0 & 3 & 1 & 38\end{array}$

$\begin{array}{lllllllllllllllllll}0 & 0 & 0 & 0 & 0 & 3 & 0 & 0 & 0 & 0 & 1 & 0 & 0 & 0 & 0 & 0 & 0 & 0 & 4\end{array}$

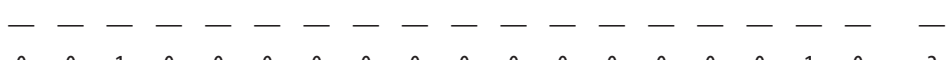

$\begin{array}{lllllllllllllllllll}0 & 0 & 1 & 0 & 0 & 0 & 0 & 0 & 0 & 0 & 0 & 0 & 0 & 0 & 0 & 0 & 1 & 0 & 2\end{array}$

$\begin{array}{lllllllllllllllllll}1 & 0 & 0 & 0 & 0 & 0 & 0 & 1 & 0 & 0 & 1 & 0 & 0 & 1 & 2 & 0 & 0 & 0 & 6\end{array}$

$\begin{array}{lllllllllllllllllll}5 & 7 & 18 & 6 & 51 & 0 & 11 & 3 & 3 & 7 & 2 & 3 & 1 & 10 & 2 & 0 & 2 & 1 & 132\end{array}$

$\begin{array}{lllllllllllllllllll}0 & 1 & 0 & 0 & 0 & 0 & 0 & 0 & 0 & 0 & 0 & 0 & 0 & 0 & 0 & 0 & 0 & 0 & 1\end{array}$

$-----\frac{-}{-}--\frac{-}{0}--\frac{-}{7}-$

$\begin{array}{lllllllllllllllllll}4 & 6 & 18 & 7 & 4 & 1 & 0 & 0 & 0 & 0 & 0 & 0 & 0 & 1 & 0 & 0 & 0 & 0 & 41\end{array}$

$\begin{array}{lllllllllllllllllll}1 & 3 & 1 & 2 & 1 & 1 & 3 & 0 & 0 & 0 & 0 & 0 & 0 & 0 & 0 & 0 & 0 & 0 & 12\end{array}$

$\begin{array}{lllllllllllllllllll}5 & 18 & 35 & 12 & 10 & 0 & 5 & 0 & 0 & 1 & 0 & 0 & 1 & 0 & 1 & 0 & 0 & 0 & 88\end{array}$

$\begin{array}{lllllllllllllllllll}0 & 0 & 0 & 0 & 0 & 0 & 0 & 0 & 0 & 0 & 0 & 0 & 0 & 0 & 2 & 0 & 0 & 0 & 2\end{array}$

$\begin{array}{lllllllllllllllllll}0 & 0 & 0 & 0 & 0 & 8 & 12 & 3 & 3 & 1 & 6 & 12 & 2 & 1 & 8 & 1 & 1 & 1 & 59\end{array}$

$\begin{array}{lllllllllllllllllll}0 & 0 & 0 & 0 & 0 & 2 & 0 & 0 & 0 & 0 & 0 & 0 & 0 & 0 & 0 & 0 & 0 & 0 & 2\end{array}$

$\begin{array}{lllllllllllllllllll}0 & 0 & 1 & 0 & 0 & 0 & 0 & 0 & 0 & 0 & 0 & 1 & 0 & 0 & 0 & 0 & 0 & 0 & 2\end{array}$

$\begin{array}{lllllllllllllllllll}3 & 3 & 0 & 1 & 0 & 2 & 1 & 3 & 4 & 0 & 0 & 0 & 2 & 0 & 1 & 0 & 0 & 0 & 20\end{array}$

$\begin{array}{lllllllllllllllllll}1 & 0 & 1 & 0 & 1 & 1 & 1 & 1 & 2 & 2 & 0 & 0 & 2 & 1 & 0 & 0 & 0 & 0 & 13\end{array}$

$\begin{array}{lllllllllllllllllll}0 & 0 & 0 & 0 & 0 & 0 & 0 & 1 & 0 & 0 & 0 & 0 & 0 & 0 & 0 & 0 & 0 & 0 & 1\end{array}$

$\begin{array}{lllllllllllllllllll}0 & 0 & 2 & 2 & 0 & 0 & 0 & 1 & 0 & 0 & 0 & 0 & 0 & 0 & 0 & 0 & 0 & 0 & 5\end{array}$

$\begin{array}{lllllllllllllllllll}0 & 1 & 6 & 0 & 2 & 2 & 16 & 6 & 3 & 0 & 8 & 4 & 1 & 0 & 11 & 0 & 2 & 0 & 62\end{array}$

$\begin{array}{lllllllllllllllllll}0 & 0 & 0 & 0 & 0 & 2 & 0 & 1 & 0 & 0 & 1 & 2 & 3 & 2 & 6 & 0 & 0 & 0 & 17\end{array}$

$\begin{array}{lllllllllllllllllll}0 & 0 & 0 & 0 & 0 & 3 & 5 & 2 & 2 & 0 & 6 & 14 & 12 & 11 & 10 & 12 & 4 & 1 & 82\end{array}$

$\begin{array}{lllllllllllllllllll}9 & 15 & 12 & 5 & 6 & 2 & 0 & 16 & 4 & 1 & 0 & 1 & 5 & 2 & 0 & 0 & 2 & 5 & 85\end{array}$

$\begin{array}{lllllllllllllllllll}0 & 0 & 11 & 18 & 49 & 0 & 0 & 2 & 0 & 0 & 0 & 0 & 0 & 0 & 0 & 0 & 0 & 0 & 80\end{array}$

$\begin{array}{lllllllllllllllllll}17 & 13 & 13 & 16 & 6 & 7 & 0 & 0 & 7 & 7 & 0 & 0 & 6 & 7 & 6 & 0 & 3 & 7 & 115\end{array}$

$\begin{array}{lllllllllllllllllll}1 & 2 & 2 & 1 & 2 & 1 & 2 & 2 & 3 & 1 & 0 & 6 & 0 & 1 & 0 & 0 & 1 & 0 & 25\end{array}$

$\begin{array}{lllllllllllllllllll}0 & 0 & 0 & 0 & 0 & 0 & 0 & 0 & 1 & 0 & 0 & 0 & 0 & 0 & 0 & 0 & 0 & 0 & \end{array}$

$\begin{array}{lllllllllllllllllll}0 & 0 & 0 & 0 & 0 & 1 & 0 & 0 & 1 & 0 & 3 & 6 & 2 & 1 & 3 & 0 & 0 & 0 & 17\end{array}$

$\begin{array}{lllllllllllllllllll}0 & 0 & 1 & 0 & 2 & 2 & 0 & 13 & 2 & 4 & 0 & 1 & 2 & 0 & 0 & 0 & 0 & 1 & 28\end{array}$

$\begin{array}{lllllllllllllllllll}0 & 0 & 0 & 0 & 0 & 0 & 7 & 7 & 0 & 1 & 1 & 2 & 2 & 0 & 0 & 4 & 2 & 0 & 26\end{array}$

$\begin{array}{lllllllllllllllllll}0 & 0 & 0 & 1 & 0 & 1 & 0 & 0 & 2 & 1 & 0 & 0 & 0 & 0 & 0 & 0 & 0 & 0 & 5\end{array}$

- - - - - - - - - - - - - -

$\begin{array}{lllllllllllllllllll}0 & 2 & 1 & 1 & 0 & 1 & 0 & 0 & 0 & 0 & 0 & 0 & 0 & 0 & 0 & 0 & 1 & 0 & 6\end{array}$

$\begin{array}{lllllllllllllllllll}2 & 18 & 5 & 3 & 2 & 0 & 2 & 9 & 0 & 1 & 1 & 0 & 0 & 0 & 0 & 0 & 1 & 0 & 44\end{array}$

$\begin{array}{lllllllllllllllllll}2 & 0 & 3 & 0 & 4 & 0 & 0 & 0 & 1 & 1 & 1 & 0 & 0 & 0 & 0 & 0 & 1 & 2 & 15\end{array}$

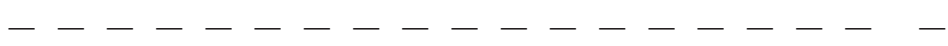

$\begin{array}{lllllllllllllllllll}0 & 0 & 1 & 0 & 0 & 0 & 1 & 0 & 0 & 1 & 1 & 2 & 0 & 1 & 1 & 0 & 0 & 0 & 8\end{array}$

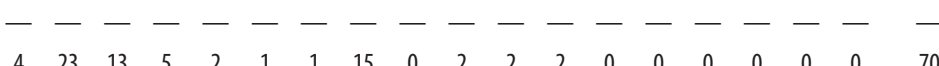

$\begin{array}{lllllllllllllllllll}0 & 0 & 0 & 0 & 0 & 0 & 0 & 0 & 1 & 0 & 0 & 0 & 0 & 0 & 0 & 0 & 1 & 1 & 3\end{array}$

$\begin{array}{lllllllllllllllllll}1 & 1 & 2 & 0 & 2 & 7 & 2 & 3 & 13 & 1 & 3 & 3 & 2 & 1 & 6 & 5 & 6 & 1 & 59\end{array}$

$\begin{array}{lllllllllllllllllll}0 & 0 & 0 & 0 & 0 & 2 & 0 & 0 & 0 & 1 & 0 & 1 & 0 & 0 & 0 & 0 & 0 & 0 & 4\end{array}$

$\begin{array}{lllllllllllllllllll}1 & 1 & 0 & 0 & 2 & 0 & 0 & 0 & 4 & 0 & 0 & 0 & 0 & 0 & 0 & 0 & 3 & 1 & 12\end{array}$

$\begin{array}{lllllllllllllllllll}1 & 0 & 0 & 0 & 0 & 1 & 0 & 0 & 4 & 1 & 0 & 1 & 0 & 1 & 5 & 1 & 0 & 1 & 16\end{array}$

$\begin{array}{lllllllllllllllllll}1 & 0 & 0 & 0 & 0 & 0 & 1 & 0 & 0 & 1 & 0 & 1 & 1 & 0 & 3 & 0 & 0 & 0 & 8\end{array}$

$\begin{array}{lllllllllllllllllll}0 & 2 & 0 & 0 & 0 & 0 & 0 & 0 & 1 & 0 & 0 & 0 & 2 & 1 & 0 & 1 & 2 & 0 & 9\end{array}$

$\begin{array}{lllllllllllllllllll}0 & 1 & 2 & 0 & 0 & 0 & 1 & 1 & 1 & 0 & 0 & 2 & 0 & 0 & 0 & 2 & 1 & 2 & 13\end{array}$

$\begin{array}{lllllllllllllllllll}0 & 0 & 0 & 0 & 0 & 0 & 0 & 0 & 1 & 0 & 0 & 0 & 0 & 0 & 0 & 0 & 0 & 0 & 1\end{array}$

$\begin{array}{lllllllllllllllllll}0 & 0 & 0 & 0 & 0 & 0 & 0 & 0 & 0 & 0 & 0 & 0 & 1 & 0 & 0 & 0 & 0 & 0 & 1\end{array}$

$\begin{array}{lllllllllllllllllll}0 & 0 & 0 & 0 & 0 & 0 & 0 & 0 & 3 & 0 & 1 & 0 & 0 & 0 & 0 & 0 & 0 & 0 & 4\end{array}$

$\begin{array}{lllllllllllllllllll}1 & 0 & 0 & 0 & 0 & 0 & 0 & 0 & 0 & 0 & 0 & 0 & 0 & 0 & 0 & 0 & 0 & 1 & 2\end{array}$

$\begin{array}{lllllllllllllllllll}0 & 0 & 0 & 0 & 1 & 0 & 0 & 0 & 0 & 0 & 0 & 0 & 0 & 0 & 0 & 0 & 0 & 0 & 1\end{array}$

$\begin{array}{lllllllllllllllllll}0 & 0 & 0 & 0 & 0 & 0 & 0 & 0 & 0 & 7 & 0 & 8 & 2 & 4 & 0 & 0 & 0 & 0 & 21\end{array}$

$\begin{array}{lllllllllllllllllll}0 & 0 & 0 & 0 & 0 & 1 & 14 & 5 & 3 & 1 & 3 & 6 & 1 & 0 & 0 & 3 & 5 & 0 & 42\end{array}$ 
FAMILY/Subfamily/Tribe/Specie

165 Viola violella (Mabille, 1898)

Erynnini

166 Anastrus sempiternus simplicior (Möschler, 1877)

167 Chiomara mithrax (Möschler, 1879)

168 Cycloglypha stellita (Zikán, 1938)

169 (ycloglypha thrasibulus thrasibulus (Fabricius, 1793)

170 Ebrietas anacreon anacreon (Staudinger, 1876)

171 Gorgythion begga begga (Prittwitz, 1868)

172 Helias phalaenoides palpalis (Latreille, [1824])

173 Mylon maimon (Fabricius, 1775)

174 Sostrata bifasciata bifasciata (Ménétriés, 1829)

175 Theagenes dichrous (Mabille, 1878)

Pyrgini

176 Antigonus liborius areta Evans, 1953

177 Antigonus minor Mielke, 1980

178 Burnsius orcus (Stoll, [1780])

179 Burnsius orcynoides (Giacomelli, 1928)

180 Carrhenes canescens pallida Röber, 1925

181 Heliopetes alana (Reakirt, 1868)

182 Heliopetes arsalte (Linnaeus, 1758)

183 Heliopetes omrina (Butler, 1870)

184 Trina geometrina geometrina (C. \& R. Felder, [1867])

185 Xenophanes tryxus (Stoll, [1780])

\section{Pyrrhopyginae}

186 *Microceris adonis (E. Bell, 1931)

187 Mysoria barcastus barta Evans, 1951

$188 *$ Oxinetra roscius roscius (Hopffer, 1874)

189 * Pyrrhopyge charybdis charybdis Westwood, 1852

\section{Tagiadinae}

Celaenorrhinini

190 *Celaenorrhinus similis Hayward, 1933

191 Celaenorrhinus eligius punctiger (Burmeister, 1878)

\section{LYCAENIDAE}

\section{Polyommatinae}

1 Leptotes cassius cassius (Cramer, [1775])

2 Zizula cyna (Edwards, 1881)

\section{Theclinae}

Eumaeini

3 Allosmaitia strophius (Godart, [1824])

4 Arawacus dolylas (Cramer, [1777])

5 Arawacus ellida (Hewitson, 1867)

6 Arawacus meliboeus (Fabricius, 1793)

7 Arawacus tadita (Hewitson, 1877)

8 Arcas ducalis (Westwood, 1852)

9 Arzecla nubilum (H. Druce, 1907)

10 Atlides atys (Cramer, [1779])

11 Brevianta celelata (Hewitson, 1874)

12 Calycopis caulonia (Hewitson, 1877)

13 Chalybs chloris (Hewitson, 1877)

14 Contrafacia catharina (Draudt, 1920)

15 Contrafacia imma (Prittwitz, 1865)

16 Cyanophrys acaste (Prittwitz, 1865)

17 Cyanophrys bertha (Jones, 1912)

18 Cyanophrys herodotus (Fabricius, 1793)

19 Cyanophrys remus (Hewitson, 1868)

20 Dicya dicaea (Hewitson, 1874)

21 Dicya eumorpha (Hayward, 1949)

22 Enos thara (Hewitson, 1867)

23 Erora biblia (Hewitson, 1868)

24 Erora campa (Jones, 1912)

$\begin{array}{lllllllllllllllllll}\text { A1 } & \text { A2 } & \text { A3 } & \text { A4 } & \text { A5 } & \text { A6 } & \text { A7 } & \text { A8 } & \text { A9 } & \text { B1 } & \text { B2 } & \text { B3 } & \text { B4 } & \text { B5 } & \text { C1 } & \text { C2 } & \text { C3 } & \text { C4 } & \text { Total }\end{array}$

$\begin{array}{ccccccccccccccccccc}\text { A1 } & \text { A2 } & \text { A3 } & \text { A4 } & \text { A5 } & \text { A6 } & \text { A7 } & \text { A8 } & \text { A9 } & \text { B1 } & \text { B2 } & \text { B3 } & \text { B4 } & \text { B5 } & \text { C1 } & \text { C2 } & \text { C3 } & \text { C4 } & \text { Total } \\ 0 & 0 & 0 & 0 & 0 & 0 & 1 & 0 & 0 & 0 & 0 & 0 & 0 & 0 & 9 & 2 & 1 & 0 & \end{array}$

$\begin{array}{llllllllllllllllllll}0 & 0 & 0 & 0 & 0 & 0 & 0 & 0 & 1 & 0 & 1 & 0 & 1 & 0 & 0 & 0 & 0 & 0 & 3\end{array}$

$\begin{array}{lllllllllllllllllll}0 & 0 & 0 & 0 & 0 & 0 & 0 & 0 & 0 & 0 & 0 & 0 & 2 & 0 & 0 & 0 & 0 & 0 & 2\end{array}$

$\begin{array}{lllllllllllllllllll}0 & 0 & 0 & 0 & 0 & 0 & 0 & 0 & 3 & 0 & 0 & 0 & 0 & 0 & 0 & 0 & 0 & 0 & 3\end{array}$

$\begin{array}{lllllllllllllllllll}0 & 0 & 0 & 0 & 0 & 2 & 0 & 0 & 2 & 0 & 0 & 0 & 0 & 0 & 0 & 0 & 1 & 0 & 5\end{array}$

$\begin{array}{lllllllllllllllllll}0 & 0 & 0 & 0 & 0 & 0 & 0 & 0 & 1 & 0 & 0 & 0 & 0 & 0 & 1 & 0 & 0 & 0 & 2\end{array}$

$\begin{array}{lllllllllllllllllll}2 & 0 & 0 & 1 & 0 & 0 & 4 & 2 & 1 & 0 & 4 & 7 & 1 & 1 & 7 & 5 & 6 & 3 & 44\end{array}$

$\begin{array}{lllllllllllllllllll}0 & 0 & 0 & 0 & 0 & 1 & 1 & 1 & 0 & 0 & 1 & 4 & 0 & 2 & 0 & 1 & 1 & 0 & 12\end{array}$

$\begin{array}{lllllllllllllllllll}0 & 0 & 0 & 0 & 0 & 2 & 0 & 0 & 0 & 0 & 0 & 3 & 2 & 1 & 1 & 0 & 0 & 0 & 9\end{array}$

$\begin{array}{lllllllllllllllllll}1 & 4 & 4 & 0 & 0 & 2 & 0 & 2 & 3 & 1 & 1 & 0 & 0 & 1 & 2 & 0 & 3 & 0 & 24\end{array}$

$\begin{array}{lllllllllllllllllll}0 & 0 & 0 & 0 & 0 & 1 & 0 & 0 & 0 & 0 & 0 & 2 & 0 & 0 & 1 & 0 & 0 & 0 & 4\end{array}$

$\begin{array}{lllllllllllllllllll}0 & 0 & 0 & 0 & 0 & 0 & 0 & 0 & 0 & 0 & 0 & 0 & 0 & 0 & 1 & 0 & 0 & 0 & 1\end{array}$

$\begin{array}{lllllllllllllllllll}0 & 0 & 0 & 0 & 0 & 0 & 0 & 0 & 0 & 0 & 0 & 1 & 0 & 0 & 0 & 0 & 0 & 0 & 1\end{array}$

$\begin{array}{lllllllllllllllllll}0 & 0 & 0 & 0 & 0 & 1 & 4 & 9 & 1 & 0 & 12 & 9 & 3 & 1 & 12 & 21 & 12 & 0 & 85\end{array}$

$\begin{array}{lllllllllllllllllll}0 & 0 & 0 & 0 & 0 & 0 & 0 & 0 & 0 & 0 & 0 & 1 & 0 & 0 & 2 & 3 & 0 & 0 & 6\end{array}$

$\begin{array}{lllllllllllllllllll}5 & 4 & 3 & 5 & 1 & 1 & 1 & 2 & 3 & 0 & 0 & 2 & 0 & 0 & 2 & 3 & 1 & 1 & 34\end{array}$

$\begin{array}{lllllllllllllllllll}0 & 0 & 0 & 0 & 0 & 1 & 2 & 2 & 2 & 0 & 1 & 6 & 2 & 0 & 1 & 0 & 2 & 0 & 19\end{array}$

$\begin{array}{lllllllllllllllllll}0 & 0 & 0 & 0 & 0 & 0 & 0 & 0 & 0 & 0 & 0 & 0 & 0 & 0 & 4 & 0 & 0 & 0 & 4\end{array}$

$\begin{array}{lllllllllllllllllll}0 & 0 & 0 & 0 & 0 & 2 & 1 & 1 & 1 & 0 & 0 & 1 & 0 & 1 & 10 & 2 & 1 & 0 & 20\end{array}$

$\begin{array}{lllllllllllllllllll}0 & 0 & 0 & 0 & 1 & 2 & 8 & 2 & 1 & 0 & 5 & 0 & 1 & 1 & 1 & 1 & 7 & 0 & 30\end{array}$

$\begin{array}{lllllllllllllllllll}0 & 0 & 0 & 0 & 0 & 0 & 12 & 3 & 2 & 0 & 0 & 4 & 0 & 0 & 3 & 1 & 1 & 0 & 26\end{array}$

$--------------$

$\begin{array}{lllllllllllllllllll}0 & 0 & 0 & 0 & 0 & 1 & 0 & 0 & 0 & 0 & 0 & 0 & 0 & 0 & 1 & 0 & 0 & 0 & 2\end{array}$

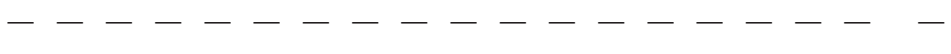

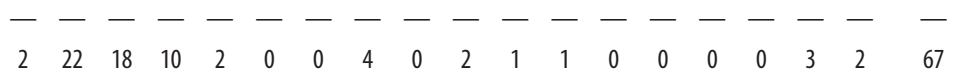
$\begin{array}{lllllllllllllllllll}0 & 0 & 0 & 0 & 0 & 2 & 0 & 3 & 0 & 0 & 0 & 6 & 3 & 0 & 3 & 0 & 1 & 0 & 18\end{array}$ $\begin{array}{lllllllllllllllllll}0 & 0 & 0 & 0 & 0 & 0 & 2 & 0 & 0 & 0 & 2 & 5 & 0 & 0 & 3 & 0 & 0 & 0 & 12\end{array}$ 
FAMILY/Subfamily/Tribe/Specie

$\begin{array}{lllllllllllllllllll}\text { A1 } & \text { A2 } & \text { A3 } & \text { A4 } & \text { A5 } & \text { A6 } & \text { A7 } & \text { A8 } & \text { A9 } & \text { B1 } & \text { B2 } & \text { B3 } & \text { B4 } & \text { B5 } & \text { C1 } & \text { C2 } & \text { C3 } & \text { C4 } & \text { Total }\end{array}$

25 Erora gabina (Godman \& Salvin, [1887])

26 Erora aff campa

27 Kolana ligurina (Hewitson, 1874)

28 Kolanasp. n.

29 Laothus phydela (Hewitson, 1867)

30 Magnastigma hirsuta (Prittwitz, 1865)

31 Ministrymon cruenta (Gosse, 1880)

32 Ministrymon azia (Hewitson, 1873)

33 Mithras catrea (Hewitson, 1874)

34 Nicolaea cupa (Druce, 1907)

35 Ocaria ocrisia (Hewitson, 1868)

$36{ }^{*}$ Ocaria sp. n.

37 Ocaria thales (Fabricius, 1793)

38 Ostrinotes sophocles (Fabricius, 1793)

39 Panthiades hebraeus (Hewitson, 1867)

40 Parrhasius orgia (Hewitson, 1867)

41 Parrhasius polibetes (Stoll, [1781])

42 Parrhasius selika (Hewitson, 1874)

43 Pseudolycaena marsyas (Linnaeus, 1758)

44 Rekoa malina (Hewitson, 1867)

45 Rekoa palegon (Cramer, [1780])

46 Siderus eliatha (Hewitson, 1867)

47 Strephonota elika (Hewitson, 1867)

48 Strymon bazochii (Godart, [1824])

49 Strymon eurytulus (Hübner, [1819])

50 Strymon oreala (Hewitson, 1868)

51 Thereus cithonius (Godart, [1824])

52 Theritas chaluma (Schaus, 1902)

53 Theritas deniva (Hewitson, 1874)

54 Theritas triquetra (Hewitson, 1865)

55 Tmolus echion (Linnaeus, 1767)

\begin{tabular}{|c|c|c|c|c|c|c|c|c|c|c|c|c|c|c|c|c|c|c|}
\hline 0 & 0 & 0 & 0 & 0 & 2 & 0 & 0 & 0 & 0 & 0 & 0 & 1 & 1 & 0 & 1 & 0 & 0 & 5 \\
\hline 0 & 0 & 0 & 0 & 0 & 1 & 0 & 0 & 0 & 0 & 0 & 0 & 0 & 0 & 0 & 0 & 0 & 0 & 1 \\
\hline 0 & 0 & 0 & 0 & 0 & 1 & 0 & 0 & 0 & 0 & 0 & 0 & 0 & 0 & 0 & 0 & 0 & 0 & 1 \\
\hline 0 & 0 & 0 & 0 & 0 & 1 & 1 & 0 & 1 & 0 & 0 & 0 & 0 & 0 & 0 & 0 & 0 & 0 & 3 \\
\hline 0 & 0 & 0 & 2 & 0 & 1 & 1 & 0 & 2 & 0 & 2 & 0 & 1 & 0 & 4 & 4 & 2 & 2 & 21 \\
\hline 0 & 0 & 0 & 0 & 0 & 1 & 0 & 0 & 1 & 0 & 1 & 0 & 0 & 1 & 0 & 0 & 0 & 0 & 4 \\
\hline 0 & 0 & 0 & 0 & 0 & 1 & 0 & 0 & 0 & 0 & 0 & 0 & 0 & 2 & 0 & 1 & 0 & 0 & 4 \\
\hline 0 & 0 & 0 & 0 & 0 & 0 & 0 & 0 & 0 & 1 & 0 & 1 & 1 & 1 & 0 & 0 & 0 & 2 & 6 \\
\hline 0 & 0 & 0 & 0 & 1 & 0 & 0 & 0 & 0 & 0 & 0 & 0 & 0 & 0 & 0 & 0 & 0 & 0 & 1 \\
\hline 0 & 0 & 0 & 0 & 1 & 0 & 0 & 2 & 1 & 0 & 0 & 0 & 0 & 0 & 0 & 0 & 0 & 1 & 5 \\
\hline 0 & 0 & 0 & 0 & 0 & 1 & 1 & 0 & 2 & 0 & 0 & 0 & 0 & 0 & 2 & 1 & 0 & 0 & 7 \\
\hline - & - & - & - & - & - & - & - & - & - & - & - & - & - & - & - & - & - & - \\
\hline 2 & 2 & 0 & 1 & 0 & 0 & 0 & 1 & 7 & 0 & 1 & 0 & 0 & 0 & 1 & 0 & 0 & 0 & 15 \\
\hline 2 & 0 & 0 & 0 & 0 & 1 & 0 & 1 & 0 & 0 & 0 & 0 & 1 & 2 & 0 & 1 & 1 & 1 & 10 \\
\hline 0 & 0 & 0 & 0 & 0 & 2 & 0 & 0 & 0 & 0 & 2 & 1 & 0 & 1 & 0 & 0 & 0 & 0 & 6 \\
\hline 0 & 0 & 0 & 0 & 1 & 0 & 0 & 0 & 2 & 0 & 0 & 0 & 0 & 1 & 5 & 0 & 1 & 0 & 10 \\
\hline 0 & 0 & 0 & 0 & 0 & 3 & 0 & 0 & 0 & 0 & 1 & 1 & 0 & 0 & 2 & 0 & 0 & 0 & 7 \\
\hline 0 & 0 & 0 & 0 & 0 & 0 & 0 & 0 & 9 & 0 & 0 & 0 & 0 & 0 & 0 & 0 & 0 & 0 & 9 \\
\hline 0 & 0 & 0 & 0 & 0 & 1 & 0 & 0 & 0 & 0 & 0 & 0 & 0 & 1 & 0 & 0 & 0 & 0 & 2 \\
\hline 0 & 0 & 0 & 0 & 0 & 7 & 0 & 0 & 2 & 0 & 0 & 1 & 0 & 0 & 3 & 2 & 0 & 0 & 15 \\
\hline 0 & 0 & 0 & 0 & 0 & 1 & 0 & 0 & 1 & 1 & 0 & 0 & 0 & 0 & 2 & 0 & 0 & 0 & 5 \\
\hline 0 & 0 & 0 & 0 & 0 & 0 & 0 & 0 & 0 & 4 & 0 & 0 & 0 & 0 & 0 & 0 & 1 & 0 & 5 \\
\hline 0 & 1 & 2 & 1 & 0 & 0 & 0 & 0 & 0 & 6 & 0 & 1 & 0 & 0 & 2 & 2 & 0 & 10 & 25 \\
\hline 0 & 0 & 0 & 0 & 0 & 0 & 0 & 0 & 0 & 0 & 0 & 1 & 0 & 0 & 2 & 0 & 0 & 0 & 3 \\
\hline 0 & 0 & 0 & 1 & 0 & 1 & 0 & 1 & 4 & 0 & 0 & 2 & 1 & 0 & 1 & 0 & 0 & 0 & 11 \\
\hline 0 & 0 & 0 & 0 & 0 & 0 & 0 & 0 & 0 & 0 & 0 & 0 & 0 & 0 & 1 & 0 & 0 & 0 & 1 \\
\hline 0 & 0 & 0 & 0 & 0 & 1 & 0 & 0 & 1 & 0 & 0 & 0 & 0 & 0 & 0 & 0 & 0 & 0 & 2 \\
\hline 0 & 0 & 0 & 0 & 0 & 0 & 0 & 0 & 0 & 0 & 0 & 0 & 0 & 0 & 1 & 0 & 0 & 0 & 1 \\
\hline 3 & 1 & 0 & 0 & 1 & 9 & 0 & 0 & 3 & 2 & 0 & 0 & 0 & 0 & 4 & 2 & 2 & 0 & 27 \\
\hline 0 & 0 & 0 & 0 & 0 & 1 & 0 & 0 & 0 & 0 & 0 & 0 & 0 & 0 & 0 & 0 & 0 & 0 & 1 \\
\hline 0 & 0 & 0 & 0 & 0 & 0 & 1 & 0 & 0 & 0 & 0 & 1 & 1 & 0 & 0 & 0 & 0 & 0 & 3 \\
\hline
\end{tabular}

\section{NYMPHALIDAE}

\section{Apaturinae}

1 Doxocopa kallina (Staudinger, 1886)

2 Doxocopa laurentia laurentia (Godart, [1824])

3 Doxocopa zunilda zunilda (Godart, [1824])

\section{Danainae}

Danaini

4 Lycorea ilione ilione (Cramer, [1775])

5 Danaus erippus (Cramer, [1775])

6 Danaus gilippus gilippus (Cramer, [1775])

Ithomiini

7 Aeria olena olena Weymer, 1875

8 Dircenna dero dero (Hübner, [1823])

9 Episcada carcinia Schaus, 1902

10 Episcada hymenaea hymenaea (Prittwitz, 1865)

11 Epityches eupompe (Geyer, 1832)

12 Hypoleria adasa adasa (Hewitson, 1855)

13 Hypothyris euclea laphria (Doubleday, [1847])

14 Ithomia agnosia zikani d'Almeida, 1940

15 Ithomia drymo Hübner, 1816

16 Mechanitis lysimnia lysimnia (Fabricius, 1793)

17 Methona themisto (Hübner, 1818)

18 Placidina euryanassa (C. Felder \& R. Felder, 1860)

19 Pseudoscada erruca (Hewitson, 1855)

20 Pteronymia sylvo (Geyer, 1832)

21 Thyridia psidii cetoides (Rosenberg \& Talbot, 1914)

\section{Biblidinae}

Ageroniinni

22 Ectima thecla thecla (Fabricius, 1796) $\begin{array}{lllllllllllllllllll}0 & 0 & 0 & 0 & 0 & 0 & 1 & 1 & 4 & 0 & 0 & 2 & 0 & 1 & 1 & 3 & 1 & 1 & 15\end{array}$

$\begin{array}{lllllllllllllllllll}0 & 0 & 2 & 1 & 1 & 2 & 2 & 5 & 7 & 1 & 1 & 1 & 1 & 1 & 6 & 13 & 4 & 3 & 51\end{array}$

$\begin{array}{lllllllllllllllllll}0 & 0 & 0 & 0 & 0 & 1 & 0 & 0 & 0 & 0 & 0 & 0 & 0 & 0 & 0 & 2 & 1 & 0 & 4\end{array}$

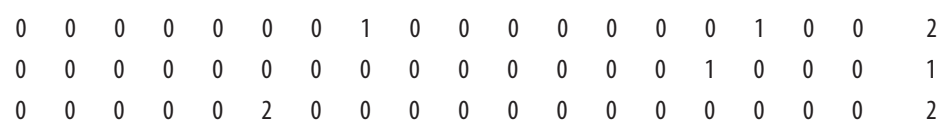

$\begin{array}{lllllllllllllllllll}0 & 0 & 0 & 0 & 0 & 0 & 0 & 0 & 0 & 0 & 0 & 0 & 0 & 0 & 0 & 0 & 1 & 0 & 1\end{array}$

$\begin{array}{lllllllllllllllllll}0 & 1 & 0 & 2 & 1 & 4 & 1 & 2 & 2 & 6 & 2 & 0 & 0 & 1 & 3 & 5 & 1 & 11 & 42\end{array}$

$\begin{array}{lllllllllllllllllll}2 & 7 & 11 & 6 & 2 & 0 & 0 & 0 & 1 & 2 & 2 & 0 & 1 & 0 & 1 & 5 & 0 & 19 & 59\end{array}$

$\begin{array}{lllllllllllllllllll}1 & 1 & 2 & 1 & 1 & 1 & 0 & 0 & 0 & 1 & 0 & 0 & 0 & 0 & 2 & 4 & 3 & 7 & 24\end{array}$

$\begin{array}{lllllllllllllllllll}8 & 9 & 12 & 8 & 4 & 8 & 2 & 7 & 1 & 9 & 5 & 1 & 1 & 5 & 3 & 13 & 4 & 19 & 119\end{array}$

$\begin{array}{lllllllllllllllllll}6 & 13 & 11 & 10 & 0 & 0 & 1 & 2 & 0 & 15 & 1 & 0 & 1 & 3 & 2 & 0 & 4 & 20 & 89\end{array}$

$\begin{array}{lllllllllllllllllll}0 & 2 & 4 & 2 & 0 & 0 & 0 & 0 & 0 & 1 & 0 & 0 & 0 & 0 & 5 & 1 & 1 & 8 & 24\end{array}$

$\begin{array}{lllllllllllllllllll}1 & 1 & 0 & 0 & 0 & 0 & 0 & 1 & 0 & 1 & 0 & 0 & 1 & 0 & 0 & 0 & 0 & 2 & 7\end{array}$

$\begin{array}{lllllllllllllllllll}1 & 0 & 0 & 0 & 0 & 0 & 0 & 2 & 0 & 3 & 0 & 0 & 1 & 2 & 0 & 1 & 0 & 3 & 13\end{array}$

$\begin{array}{lllllllllllllllllll}6 & 1 & 1 & 1 & 1 & 0 & 5 & 1 & 1 & 2 & 2 & 0 & 1 & 3 & 5 & 2 & 3 & 11 & 46\end{array}$

$\begin{array}{lllllllllllllllllll}0 & 0 & 2 & 1 & 0 & 0 & 0 & 0 & 0 & 1 & 0 & 0 & 2 & 0 & 0 & 0 & 0 & 0 & 6\end{array}$

$\begin{array}{lllllllllllllllllll}0 & 0 & 0 & 0 & 0 & 0 & 0 & 1 & 0 & 0 & 0 & 0 & 0 & 0 & 1 & 0 & 0 & 0 & 2\end{array}$

$\begin{array}{lllllllllllllllllll}3 & 12 & 24 & 7 & 2 & 0 & 2 & 1 & 0 & 10 & 1 & 1 & 1 & 0 & 4 & 2 & 2 & 20 & 92\end{array}$

$\begin{array}{lllllllllllllllllll}2 & 3 & 2 & 0 & 0 & 0 & 0 & 0 & 0 & 1 & 0 & 0 & 0 & 0 & 0 & 0 & 0 & 2 & 10\end{array}$

$\begin{array}{lllllllllllllllllll}0 & 0 & 0 & 0 & 0 & 0 & 0 & 0 & 0 & 0 & 0 & 1 & 0 & 0 & 0 & 0 & 1 & 0 & 2\end{array}$ 


\begin{tabular}{|c|c|c|c|c|c|c|c|c|c|c|c|c|c|c|c|c|c|c|c|}
\hline FAMILY/Subfamily/Tribe/Specie & A1 & A2 & A3 & A4 & A5 & A6 & A7 & A8 & A9 & B1 & B2 & B3 & B4 & B5 & C1 & C2 & C3 & C4 & Total \\
\hline 23 Hamadryas amphinome amphinome (Linnaeus, 1767) & 0 & 0 & 0 & 0 & 0 & 1 & 0 & 1 & 0 & 0 & 0 & 0 & 0 & 0 & 0 & 0 & 0 & 0 & 2 \\
\hline 24 Hamadryas epinome (C. \& R. Felder, 1867) & 1 & 2 & 0 & 0 & 4 & 4 & 0 & 6 & 1 & 3 & 1 & 0 & 13 & 1 & 1 & 1 & 0 & 1 & 39 \\
\hline 25 Hamadryas februa februa (Hübner, [1823]) & 0 & 0 & 0 & 0 & 1 & 0 & 0 & 0 & 0 & 0 & 0 & 0 & 0 & 0 & 0 & 0 & 0 & 0 & 1 \\
\hline $\begin{array}{l}26 \text { Hamadryas fornax fornax (Hübner, [1823]) } \\
\text { Biblidini }\end{array}$ & 0 & 0 & 0 & 0 & 0 & 0 & 0 & 0 & 0 & 1 & 0 & 0 & 2 & 0 & 0 & 0 & 0 & 0 & 3 \\
\hline $\begin{array}{l}27 \text { Biblis hyperia nectanabis (Fruhstorfer, 1909) } \\
\text { Callicorini }\end{array}$ & 5 & 0 & 2 & 1 & 1 & 6 & 6 & 7 & 4 & 4 & 10 & 4 & 5 & 3 & 2 & 1 & 7 & 1 & 69 \\
\hline 28 Callicore pygas eucale (Fruhstorfer, 1916) & 3 & 2 & 0 & 0 & 0 & 1 & 1 & 0 & 0 & 0 & 0 & 0 & 0 & 0 & 2 & 1 & 0 & 0 & 10 \\
\hline 29 Diaethria candrena candrena (Godart, [1824]) & 0 & 0 & 0 & 0 & 0 & 1 & 1 & 0 & 0 & 0 & 0 & 1 & 0 & 1 & 0 & 2 & 2 & 2 & 10 \\
\hline 30 Diaethria clymena meridionalis (Bates, 1864) & 0 & 0 & 0 & 0 & 2 & 0 & 0 & 0 & 0 & 0 & 0 & 1 & 2 & 0 & 4 & 7 & 0 & 0 & 16 \\
\hline $\begin{array}{l}31 \text { Haematera pyrame pyrame (Hübner, [1819]) } \\
\text { Catonephelini }\end{array}$ & 0 & 0 & 0 & 0 & 0 & 0 & 0 & 2 & 0 & 0 & 0 & 0 & 0 & 0 & 2 & 0 & 0 & 0 & 4 \\
\hline 32 Cybdelis phaesyla (Hübner, [1831]) & 0 & 0 & 0 & 0 & 0 & 0 & 0 & 0 & 0 & 0 & 0 & 0 & 0 & 0 & 1 & 0 & 0 & 0 & 1 \\
\hline 33 Eunica caelina caelina (Godart, [1824]) & 0 & 0 & 0 & 0 & 0 & 0 & 0 & 0 & 0 & 0 & 0 & 0 & 0 & 0 & 0 & 1 & 0 & 0 & 1 \\
\hline 34 Eunica eburnea Fruhstorfer, 1907 & 0 & 0 & 0 & 0 & 0 & 0 & 5 & 1 & 0 & 0 & 4 & 6 & 2 & 0 & 3 & 2 & 3 & 0 & 26 \\
\hline 35 Eunica tatila bellaria Fruhstorfer, 1908 & 0 & 0 & 0 & 0 & 0 & 0 & 0 & 0 & 0 & 0 & 0 & 1 & 0 & 0 & 3 & 0 & 0 & 0 & 4 \\
\hline $\begin{array}{l}36 \text { Myscelia orsis (Drury, [1782]) } \\
\text { Eubagini }\end{array}$ & 0 & 0 & 0 & 0 & 0 & 0 & 0 & 1 & 0 & 2 & 1 & 1 & 2 & 1 & 0 & 0 & 0 & 0 & 8 \\
\hline 37 Dynamine agadles agacles (Dalman, 1823) & 0 & 0 & 0 & 0 & 1 & 7 & 2 & 0 & 0 & 0 & 0 & 1 & 2 & 5 & 0 & 0 & 0 & 0 & 18 \\
\hline 38 Dynamine artemisia artemisia (Fabricius, 1793) & 1 & 1 & 0 & 0 & 0 & 0 & 1 & 1 & 0 & 0 & 1 & 0 & 0 & 0 & 0 & 0 & 0 & 0 & 5 \\
\hline 39 Dynamine athemon athemaena (Hübner, [1824]) & 0 & 0 & 1 & 0 & 0 & 0 & 0 & 0 & 0 & 0 & 1 & 3 & 2 & 3 & 0 & 0 & 0 & 0 & 10 \\
\hline 40 Dynamine coenus (Fabricius, 1793) & 0 & 0 & 0 & 0 & 0 & 0 & 0 & 0 & 4 & 1 & 0 & 0 & 0 & 0 & 0 & 0 & 0 & 0 & 5 \\
\hline 41 Dynamine myrrhina (Doubleday, 1849) & 10 & 0 & 0 & 0 & 7 & 2 & 6 & 12 & 6 & 9 & 10 & 4 & 2 & 3 & 7 & 15 & 15 & 8 & 116 \\
\hline 42 Dynamine postverta postverta (Cramer, [1780]) & 0 & 0 & 0 & 0 & 0 & 2 & 1 & 2 & 0 & 0 & 0 & 1 & 0 & 1 & 0 & 0 & 0 & 0 & 7 \\
\hline $\begin{array}{l}43 \text { Dynamine tithia tithia (Hübner, [1823]) } \\
\text { Epiphelini }\end{array}$ & 0 & 1 & 0 & 0 & 0 & 1 & 1 & 0 & 1 & 0 & 1 & 2 & 1 & 4 & 0 & 0 & 0 & 0 & 12 \\
\hline 44 Epiphile hubneri Hewitson, 1861 & 0 & 0 & 0 & 0 & 0 & 0 & 0 & 0 & 0 & 1 & 0 & 0 & 1 & 0 & 1 & 6 & 1 & 2 & 12 \\
\hline 45 Epiphile orea orea (Hübner, [1823]) & 0 & 0 & 1 & 0 & 0 & 0 & 1 & 0 & 0 & 1 & 0 & 0 & 0 & 0 & 2 & 0 & 1 & 1 & 7 \\
\hline 46 Temenis laothoe meridionalis Ebert, 1965 & 0 & 0 & 0 & 0 & 0 & 0 & 2 & 0 & 1 & 0 & 0 & 3 & 0 & 0 & 0 & 1 & 0 & 0 & 7 \\
\hline $\begin{array}{l}\text { Charaxinae } \\
\text { Anaeini }\end{array}$ & & & & & & & & & & & & & & & & & & & \\
\hline 47 Hypna clytemnestra huebneri Butler, 1866 & 0 & 0 & 0 & 0 & 0 & 0 & 0 & 0 & 0 & 0 & 0 & 0 & 0 & 0 & 1 & 0 & 0 & 0 & 1 \\
\hline 48 Memphis acidalia victoria (Druce, 1877) & 1 & 0 & 0 & 0 & 0 & 0 & 0 & 0 & 0 & 0 & 0 & 0 & 0 & 0 & 0 & 0 & 0 & 1 & 2 \\
\hline 49 Memphis moruus stheno (Prittwitz, 1865) & 0 & 0 & 0 & 1 & 0 & 0 & 0 & 0 & 0 & 0 & 0 & 0 & 0 & 1 & 1 & 0 & 0 & 0 & 3 \\
\hline 50 Memphis otrere (Hübner, 1825) & 0 & 0 & 0 & 0 & 0 & 0 & 0 & 1 & 0 & 0 & 0 & 0 & 0 & 0 & 0 & 0 & 0 & 0 & 1 \\
\hline $\begin{array}{l}51 \text { Zaretis strigosus (Gmelin, [1790]) } \\
\text { Preponini }\end{array}$ & 0 & 0 & 0 & 0 & 0 & 0 & 0 & 1 & 0 & 1 & 0 & 0 & 0 & 0 & 0 & 0 & 0 & 0 & 2 \\
\hline 52 *Archaeoprepona amphimachus pseudomeander (Fruhstorfer, 1906) & - & - & - & - & - & - & - & - & - & - & - & - & - & - & - & - & - & - & - \\
\hline 53 Archaeoprepona chalciope (Hübner, [1823]) & 2 & 0 & 0 & 0 & 0 & 0 & 0 & 0 & 0 & 0 & 0 & 0 & 0 & 0 & 0 & 0 & 0 & 0 & 2 \\
\hline 54 *Archaeoprepona demophon thalpius (Hübner, [1814]) & - & - & - & - & - & - & - & - & - & - & - & - & - & - & - & - & - & - & - \\
\hline 55 *Archaeoprepona demophoon antimache (Hübner, [1819]) & - & - & - & - & - & - & - & - & - & - & - & - & - & - & - & - & - & - & - \\
\hline $\begin{array}{l}56 \text { Prepona pylene Hewitson, } 1854 \\
\text { Cyrestinae } \\
\text { Cyrestini }\end{array}$ & 0 & 0 & 0 & 0 & 1 & 0 & 0 & 0 & 0 & 0 & 0 & 0 & 0 & 0 & 0 & 0 & 0 & 0 & 1 \\
\hline $\begin{array}{l}57 \text { * Marpesia petreus (Cramer, [1776]) } \\
\text { Heliconiinae } \\
\text { Acraeini }\end{array}$ & - & - & - & - & - & - & - & - & - & - & - & - & - & - & - & - & - & - & - \\
\hline 58 Actinote carycina Jordan, 1913 & 0 & 0 & 0 & 0 & 7 & 13 & 2 & 1 & 13 & 4 & 12 & 9 & 12 & 25 & 15 & 8 & 13 & 0 & 134 \\
\hline 59 Actinote dalmeidai Francini, 1996 & 0 & 0 & 0 & 0 & 2 & 0 & 0 & 0 & 0 & 0 & 0 & 0 & 0 & 0 & 0 & 1 & 1 & 0 & 4 \\
\hline 60 Actinote genitrix R.F. d'Almeida, 1922 & 1 & 0 & 0 & 0 & 3 & 1 & 0 & 1 & 0 & 0 & 0 & 1 & 0 & 4 & 0 & 2 & 0 & 0 & 13 \\
\hline 61 Actinote melanisans Oberthür, 1917 & 1 & 0 & 0 & 0 & 2 & 3 & 2 & 2 & 3 & 1 & 1 & 1 & 6 & 0 & 1 & 4 & 2 & 0 & 29 \\
\hline $\begin{array}{l}62 \text { Actinote surima surima (Schaus, 1902) } \\
\text { Argynnini }\end{array}$ & 0 & 0 & 0 & 0 & 0 & 2 & 0 & 0 & 0 & 0 & 5 & 0 & 0 & 3 & 2 & 0 & 0 & 0 & 12 \\
\hline $\begin{array}{l}63 \text { Euptoieta hortensia (Blanchard, 1852) } \\
\text { Heliconini }\end{array}$ & 0 & 0 & 0 & 0 & 3 & 0 & 0 & 0 & 0 & 0 & 1 & 0 & 0 & 0 & 3 & 0 & 0 & 0 & 7 \\
\hline 64 Agraulis vanillae maculosa (Stichel, [1908]) & 0 & 0 & 0 & 0 & 0 & 0 & 2 & 1 & 0 & 0 & 1 & 2 & 3 & 2 & 0 & 0 & 0 & 0 & 11 \\
\hline 65 Dione juno juno (Cramer, [1779]) & 0 & 0 & 0 & 0 & 0 & 0 & 0 & 0 & 1 & 0 & 0 & 2 & 1 & 0 & 0 & 0 & 0 & 0 & 4 \\
\hline 66 Dione moneta moneta Hübner, [1825] & 0 & 0 & 0 & 0 & 0 & 1 & 0 & 0 & 1 & 0 & 0 & 1 & 1 & 0 & 2 & 4 & 4 & 0 & 14 \\
\hline 67 Dryas iulia alcionea (Cramer, 1779) & 0 & 0 & 0 & 0 & 1 & 0 & 0 & 0 & 0 & 0 & 2 & 0 & 7 & 2 & 2 & 1 & 5 & 2 & 22 \\
\hline 68 Eueides aliphera aliphera (Godart, 1819) & 0 & 0 & 0 & 0 & 0 & 0 & 0 & 1 & 0 & 1 & 0 & 0 & 0 & 0 & 0 & 2 & 1 & 1 & 6 \\
\hline
\end{tabular}


FAMILY/Subfamily/Tribe/Specie

69 Eueides isabella dianasa (Hübner, [1806])

70 Heliconius erato phyllis (Fabricius, 1775)

71 Heliconius ethilla narcaea Godart, 1819

\section{Libytheinae}

72 Libytheana carinenta carinenta (Cramer, [1777])

\section{Limenitidinae}

Limenitidini

73 Adelpha falcipennis Fruhstorfer, 1915

74 Adelpha hyas hyas (Doyère, [1840])

75 Adelpha iphiclus ephesa (Ménétriés, 1857)

76 Adelpha mythra (Godart, [1824])

77 Adelpha serpa serpa (Boisduval, [1836])

78 Adelpha syma (Godart, [1824])

79 Adelpha thessalia indefecta Fruhstorfer, 1913

80 Adelpha zea (Hewitson, 1850)

\section{Nymphalinae}

\section{Melitaeini}

81 Chlosyne lacinia saundersi (Doubleday, [1847])

82 Eresia lansdorfi (Godart, 1819)

83 Ortilia dicoma (Hewitson, 1864)

84 Ortilia ithra (Kirby, 1900)

85 Ortilia orthia (Hewitson, 1864)

86 Ortilia velica durnfordi (Godman \& Salvin, 1878)

87 Tegosa claudina (Eschscholtz, 1821)

88 Telenassa teletusa teletusa (Godart, [1824])

$$
\text { Nymphalini }
$$

89 Hypanartia bella (Fabricius, 1793)

90 Hypanartia lethe lethe (Fabricius, 1793)

91 Smyrna blomfildia blomfildia (Fabricius, 1781)

92 Vanessa braziliensis (Moore, 1883)

93 Vanessa carye (Hübner, [1812])

94 Vanessa myrinna (Doubleday, 1849)

Junoniini

95 Junonia evarete evarete (Cramer, [1779])

\section{Victorinini}

96 Anartia amathea roeselia (Eschscholtz, 1821)

97 Siproeta epaphus trayja Hübner, [1823]

\section{Satyrinae}

Brassolini

98 Blepolenis bassus (C. \& R. Felder, [1867])

99 Blepolenis batea batea (Hübner, [1821])

100 *Caligo illioneus pampeiro Fruhstorfer, 1904

101 Caligo martia (Godart, [1824])

102 Eryphanis reevesii (Doubleday, [1849])

103 Opoptera sulcius (Staudinger, 1887)

$104{ }^{*}$ Opsiphanes cassiae crameri C. Felder \& R. Felder, 1862

$105 *$ Opsiphanes quiteria meridionalis Staudinger, 1887

106 Opsiphanes invirae amplificatus Stichel, 1904

Morphini

107 Morpho aega aega (Hübner, [1822])

108 Morpho anaxibia (Esper, 1801)

109 Morpho epistrophus catenaria (Perry, 1811)

110 Morpho helenor violaceus Fruhstorfer, 1912

Satyrini

111 Capronnieria galesus (Godart, [1824])

112 Carminda griseldis (Weymer, 1911)

113 Carminda paeon (Godart, [1824])

114 Cissia eous (Butler, 1867)

115 Cissia phronius (Godart, [1824])

116 Eteona tisiphone (Boisduval, [1836])

$\begin{array}{ccccccccccccccccccc}\text { A1 } & \text { A2 } & \text { A3 } & \text { A4 } & \text { A5 } & \text { A6 } & \text { A7 } & \text { A8 } & \text { A9 } & \text { B1 } & \text { B2 } & \text { B3 } & \text { B4 } & \text { B5 } & \text { C1 } & \text { C2 } & \text { C3 } & \text { C4 } & \text { Total } \\ 0 & 0 & 0 & 0 & 0 & 0 & 0 & 0 & 0 & 0 & 1 & 0 & 0 & 0 & 0 & 0 & 0 & 0 & 1 \\ 15 & 4 & 3 & 2 & 7 & 4 & 2 & 3 & 3 & 21 & 10 & 4 & 6 & 15 & 4 & 2 & 9 & 4 & 118 \\ 1 & 0 & 0 & 0 & 0 & 0 & 0 & 0 & 0 & 3 & 0 & 0 & 0 & 3 & 0 & 0 & 0 & 1 & 8 \\ & & & & & & & & & & & & & & & & & & \\ 0 & 0 & 0 & 0 & 0 & 1 & 0 & 0 & 1 & 0 & 0 & 0 & 1 & 1 & 1 & 6 & 2 & 0 & 13 \\ & & & & & & & & & & & & & & & & & & \\ & & & & & & & & & & & & & & & & & & \\ 0 & 0 & 0 & 0 & 0 & 0 & 0 & 0 & 2 & 0 & 0 & 0 & 0 & 0 & 0 & 2 & 0 & 0 & 4 \\ 0 & 0 & 0 & 0 & 0 & 1 & 0 & 2 & 1 & 0 & 0 & 1 & 0 & 1 & 0 & 0 & 0 & 0 & 6 \\ 0 & 0 & 0 & 0 & 0 & 0 & 0 & 0 & 1 & 0 & 0 & 0 & 0 & 0 & 0 & 0 & 0 & 0 & 1 \\ 6 & 0 & 1 & 0 & 0 & 1 & 0 & 2 & 3 & 1 & 0 & 1 & 0 & 0 & 2 & 0 & 4 & 1 & 22 \\ 0 & 0 & 0 & 0 & 0 & 0 & 1 & 0 & 1 & 1 & 1 & 1 & 0 & 0 & 0 & 0 & 0 & 0 & 5 \\ 15 & 2 & 1 & 1 & 2 & 6 & 15 & 6 & 24 & 4 & 20 & 16 & 4 & 5 & 15 & 7 & 7 & 2 & 152 \\ 6 & 0 & 1 & 1 & 0 & 7 & 0 & 4 & 15 & 0 & 0 & 0 & 0 & 1 & 2 & 6 & 4 & 1 & 48 \\ 1 & 0 & 0 & 0 & 0 & 2 & 0 & 1 & 5 & 0 & 0 & 0 & 0 & 2 & 1 & 0 & 0 & 0 & 12\end{array}$

$\begin{array}{lllllllllllllllllll}0 & 0 & 0 & 0 & 0 & 3 & 0 & 3 & 3 & 0 & 1 & 6 & 1 & 0 & 0 & 5 & 0 & 0 & 22\end{array}$

$\begin{array}{lllllllllllllllllll}2 & 2 & 0 & 2 & 0 & 2 & 1 & 0 & 0 & 0 & 1 & 2 & 1 & 4 & 0 & 1 & 1 & 1 & 20\end{array}$

$\begin{array}{lllllllllllllllllll}0 & 0 & 1 & 0 & 2 & 1 & 0 & 1 & 0 & 9 & 2 & 1 & 0 & 5 & 0 & 3 & 2 & 0 & 27\end{array}$

$\begin{array}{lllllllllllllllllll}0 & 0 & 0 & 0 & 0 & 3 & 4 & 4 & 2 & 0 & 2 & 10 & 1 & 1 & 10 & 9 & 1 & 1 & 48\end{array}$

$\begin{array}{lllllllllllllllllll}3 & 0 & 1 & 0 & 1 & 3 & 3 & 11 & 12 & 5 & 8 & 29 & 7 & 3 & 9 & 10 & 11 & 0 & 116\end{array}$

$\begin{array}{lllllllllllllllllll}0 & 0 & 0 & 0 & 0 & 1 & 0 & 0 & 0 & 0 & 0 & 0 & 0 & 0 & 0 & 0 & 0 & 0 & 1\end{array}$

$\begin{array}{lllllllllllllllllll}1 & 0 & 0 & 0 & 0 & 1 & 5 & 23 & 14 & 5 & 16 & 29 & 7 & 7 & 13 & 18 & 17 & 0 & 156\end{array}$

$\begin{array}{lllllllllllllllllll}3 & 2 & 3 & 5 & 4 & 1 & 1 & 6 & 1 & 2 & 1 & 3 & 1 & 0 & 0 & 2 & 1 & 3 & 39\end{array}$

$\begin{array}{lllllllllllllllllll}0 & 0 & 3 & 0 & 1 & 2 & 2 & 2 & 2 & 2 & 7 & 12 & 1 & 4 & 4 & 2 & 2 & 0 & 46\end{array}$

$\begin{array}{lllllllllllllllllll}0 & 0 & 1 & 0 & 2 & 4 & 2 & 5 & 2 & 0 & 1 & 0 & 6 & 0 & 1 & 7 & 6 & 1 & 38\end{array}$

$\begin{array}{lllllllllllllllllll}2 & 0 & 0 & 0 & 0 & 1 & 0 & 0 & 1 & 0 & 0 & 0 & 0 & 0 & 0 & 1 & 0 & 0 & 5\end{array}$

$\begin{array}{lllllllllllllllllll}0 & 0 & 0 & 0 & 0 & 5 & 2 & 2 & 0 & 0 & 6 & 2 & 2 & 3 & 10 & 2 & 1 & 0 & 35\end{array}$

$\begin{array}{lllllllllllllllllll}0 & 0 & 0 & 0 & 0 & 0 & 0 & 0 & 0 & 0 & 1 & 0 & 0 & 0 & 0 & 0 & 0 & 0 & 1\end{array}$

$\begin{array}{lllllllllllllllllll}0 & 0 & 0 & 0 & 0 & 2 & 2 & 1 & 0 & 0 & 0 & 0 & 1 & 1 & 0 & 0 & 0 & 0 & 7\end{array}$

$\begin{array}{lllllllllllllllllll}0 & 0 & 0 & 0 & 0 & 3 & 0 & 1 & 0 & 0 & 1 & 0 & 0 & 0 & 7 & 1 & 0 & 0 & 13\end{array}$

$\begin{array}{lllllllllllllllllll}3 & 0 & 7 & 0 & 0 & 6 & 9 & 3 & 2 & 0 & 8 & 26 & 4 & 1 & 25 & 14 & 4 & 0 & 112\end{array}$

$\begin{array}{lllllllllllllllllll}0 & 0 & 1 & 0 & 0 & 0 & 1 & 1 & 1 & 1 & 0 & 0 & 1 & 0 & 3 & 1 & 0 & 0 & 10\end{array}$ 


\begin{tabular}{|c|c|c|c|c|c|c|c|c|c|c|c|c|c|c|c|c|c|c|c|}
\hline FAMILY/Subfamily/Tribe/Specie & A1 & A2 & A3 & A4 & A5 & A6 & A7 & A8 & A9 & B1 & B2 & B3 & B4 & B5 & C1 & C2 & C3 & C4 & Total \\
\hline 117 Euptychoides castrensis (Schaus, 1902) & 5 & 1 & 1 & 0 & 0 & 0 & 0 & 0 & 4 & 0 & 0 & 0 & 0 & 0 & 0 & 0 & 0 & 3 & 14 \\
\hline 118 Forsterinaria necys (Godart, [1824]) & 0 & 0 & 1 & 3 & 2 & 0 & 0 & 0 & 1 & 7 & 0 & 0 & 0 & 0 & 1 & 0 & 0 & 1 & 16 \\
\hline 119 Forsterinaria quantius (Godart, [1824]) & 1 & 0 & 0 & 1 & 11 & 2 & 0 & 0 & 4 & 1 & 0 & 0 & 4 & 1 & 2 & 0 & 0 & 1 & 28 \\
\hline 120 Godartiana muscosa (Butler, 1870) & 16 & 22 & 24 & 39 & 3 & 0 & 0 & 0 & 2 & 18 & 0 & 0 & 3 & 6 & 4 & 2 & 4 & 17 & 160 \\
\hline 121 Guaianaza pronophila (Butler, 1867) & 0 & 0 & 0 & 0 & 0 & 0 & 0 & 0 & 0 & 0 & 0 & 0 & 0 & 0 & 0 & 0 & 1 & 0 & 1 \\
\hline 122 Hermeuptychia aff hermes sp.1 & 6 & 4 & 7 & 3 & 2 & 7 & 5 & 1 & 5 & 0 & 11 & 3 & 11 & 6 & 14 & 18 & 3 & 6 & 112 \\
\hline 123 Hermeuptychia aff hermes sp.2 & 6 & 4 & 1 & 0 & 1 & 1 & 0 & 1 & 0 & 0 & 1 & 0 & 0 & 0 & 1 & 0 & 2 & 1 & 19 \\
\hline 124 Hermeuptychia aff hermes sp.3 & 1 & 0 & 0 & 1 & 0 & 0 & 0 & 1 & 2 & 0 & 0 & 0 & 0 & 0 & 0 & 0 & 1 & 1 & 7 \\
\hline 125 Hermeuptychia sp. n. & 1 & 0 & 0 & 0 & 0 & 0 & 0 & 0 & 0 & 0 & 0 & 0 & 0 & 0 & 0 & 0 & 0 & 0 & 1 \\
\hline 126 Moneuptychia soter (Butler, 1877) & 2 & 1 & 1 & 0 & 1 & 4 & 5 & 5 & 1 & 0 & 5 & 0 & 2 & 2 & 2 & 8 & 7 & 7 & 53 \\
\hline 127 Paryphthimoides poltys (Prittwitz, 1865) & 1 & 4 & 4 & 0 & 0 & 0 & 0 & 1 & 0 & 1 & 0 & 1 & 0 & 0 & 0 & 0 & 2 & 3 & 17 \\
\hline 128 Paryphthimoides undulata (Butler, 1867) & 0 & 0 & 0 & 0 & 0 & 0 & 0 & 0 & 0 & 0 & 0 & 0 & 1 & 0 & 0 & 0 & 0 & 0 & 1 \\
\hline 129 Praepedaliodes phanias (Hewitson, 1862) & 0 & 0 & 0 & 0 & 0 & 0 & 0 & 1 & 0 & 0 & 0 & 0 & 0 & 0 & 0 & 0 & 0 & 0 & 1 \\
\hline 130 Taygetis acuta Weymer, 1910 & 0 & 0 & 0 & 1 & 0 & 0 & 0 & 0 & 0 & 0 & 0 & 0 & 0 & 0 & 0 & 0 & 0 & 0 & 1 \\
\hline 131 Taygetis ypthima Hübner, [1821] & 5 & 2 & 1 & 22 & 0 & 0 & 0 & 1 & 3 & 0 & 0 & 0 & 0 & 0 & 0 & 0 & 0 & 0 & 34 \\
\hline 132 Yphthimoides celmis (Godart, [1824]) & 0 & 0 & 0 & 0 & 0 & 0 & 0 & 0 & 0 & 0 & 0 & 0 & 2 & 0 & 0 & 0 & 0 & 0 & 2 \\
\hline 133 Yphthimoides leguialimai (Dyar, 1913) & 0 & 0 & 0 & 0 & 0 & 0 & 0 & 0 & 0 & 0 & 1 & 0 & 1 & 0 & 0 & 0 & 0 & 0 & 2 \\
\hline 134 Yphthimoidessp. n. & 0 & 0 & 0 & 0 & 0 & 1 & 6 & 0 & 0 & 0 & 8 & 0 & 0 & 0 & 0 & 0 & 0 & 0 & 15 \\
\hline 135 Yphthimoides ordinaria Freitas, Kaminski \& Mielke, 2012 & 0 & 0 & 0 & 0 & 1 & 2 & 1 & 0 & 0 & 0 & 4 & 5 & 10 & 6 & 0 & 0 & 0 & 0 & 29 \\
\hline 136 Yphthimoides renata (Stoll, [1780]) & 0 & 0 & 0 & 0 & 3 & 0 & 0 & 0 & 0 & 0 & 0 & 0 & 0 & 0 & 0 & 0 & 0 & 0 & 3 \\
\hline 137 Zischkaia pacarus (Godart, [1824]) & 1 & 0 & 0 & 0 & 4 & 2 & 1 & 1 & 2 & 0 & 0 & 0 & 3 & 1 & 4 & 0 & 0 & 0 & 19 \\
\hline
\end{tabular}

137 Zischkaia pacarus (Godart, [1824])

\begin{tabular}{|c|c|c|c|c|c|c|c|c|c|c|c|c|c|c|c|c|c|c|c|}
\hline \multicolumn{20}{|l|}{ PAPILIONIDAE } \\
\hline \multicolumn{20}{|l|}{ Papilioninae } \\
\hline \multicolumn{20}{|l|}{ Leptocircini } \\
\hline 1 Mimoides lysithous lysithous (Hübner, [1821]) & 0 & 0 & 0 & 0 & 1 & 0 & 0 & 0 & 0 & 0 & 1 & 1 & 2 & 0 & 0 & 0 & 1 & 2 & 8 \\
\hline 2 Mimoides lysithous rurik (Eschscholtz, 1821) & 0 & 0 & 0 & 1 & 1 & 4 & 1 & 2 & 1 & 0 & 0 & 0 & 1 & 1 & 0 & 0 & 1 & 2 & 15 \\
\hline 3 Protesilaus helios (Rothschild \& Jordan, 1906) & 0 & 0 & 0 & 0 & 0 & 0 & 0 & 0 & 0 & 0 & 0 & 0 & 0 & 0 & 1 & 0 & 0 & 0 & 1 \\
\hline 4 *Protesilaus protesilaus nigricornis (Staudinger, 1884) & - & - & - & - & - & - & - & - & - & - & - & - & - & - & - & - & - & - & - \\
\hline 5 Protesilaus stenodesmus (Rothschild \& Jordan, 1906) & 0 & 0 & 0 & 0 & 0 & 0 & 0 & 0 & 0 & 0 & 0 & 0 & 0 & 0 & 1 & 0 & 0 & 0 & 1 \\
\hline \multicolumn{20}{|l|}{ Troidini } \\
\hline 6 Battus polydamas polydamas (Linnaeus, 1758 & 0 & 0 & 0 & 0 & 0 & 0 & 0 & 0 & 0 & 0 & 0 & 0 & 1 & 0 & 1 & 1 & 0 & 0 & 3 \\
\hline 7 Battus polystictus polystictus (Butler, 1874) & 0 & 0 & 0 & 0 & 1 & 1 & 0 & 3 & 0 & 0 & 0 & 0 & 4 & 2 & 1 & 0 & 0 & 0 & 12 \\
\hline 8 Parides agavus (Drury, 1782) & 1 & 1 & 0 & 0 & 1 & 0 & 2 & 5 & 0 & 1 & 0 & 0 & 0 & 2 & 1 & 0 & 0 & 0 & 14 \\
\hline 9 Parides anchises nephalion (Godart, 1819) & 0 & 0 & 0 & 0 & 0 & 0 & 0 & 1 & 0 & 0 & 0 & 0 & 0 & 0 & 0 & 0 & 0 & 0 & 1 \\
\hline 10 Parides bunichus perrhebus (Boisduval, 1836) & 0 & 0 & 0 & 1 & 3 & 1 & 3 & 6 & 0 & 0 & 0 & 0 & 4 & 8 & 2 & 0 & 1 & 0 & 29 \\
\hline \multicolumn{20}{|l|}{ Papilionini } \\
\hline 11 Heraclides anchisiades capys (Huebner,. 1809) & 0 & 0 & 0 & 0 & 0 & 0 & 1 & 0 & 0 & 0 & 0 & 0 & 1 & 4 & 0 & 0 & 0 & 0 & 6 \\
\hline 12 Heraclides astyalus astyalus (Godart, 1819) & 1 & 0 & 0 & 0 & 0 & 3 & 0 & 2 & 0 & 0 & 1 & 1 & 1 & 1 & 3 & 0 & 1 & 0 & 14 \\
\hline 13 Heraclides hectorides (Esper, 1794) & 0 & 2 & 1 & 1 & 0 & 1 & 1 & 0 & 1 & 1 & 0 & 1 & 1 & 6 & 1 & 1 & 1 & 5 & 24 \\
\hline 14 Heraclides thoas brasiliensis (Rothschild \& Jordan, 1906) & 0 & 0 & 0 & 0 & 0 & 0 & 0 & 0 & 0 & 0 & 0 & 0 & 0 & 3 & 1 & 0 & 0 & 0 & 4 \\
\hline 15 Pterourus menatius cleotas (G. Gray, 1832) & 0 & 0 & 0 & 0 & 0 & 0 & 0 & 0 & 0 & 0 & 0 & 0 & 0 & 0 & 1 & 0 & 0 & 0 & 1 \\
\hline 16 Pterourus scamander scamander (Boisduval, 1836) & 0 & 0 & 0 & 0 & 0 & 2 & 0 & 0 & 0 & 0 & 1 & 0 & 0 & 0 & 6 & 0 & 0 & 0 & 9 \\
\hline \multicolumn{20}{|l|}{ PIERIDAE } \\
\hline \multicolumn{20}{|l|}{ Dismorphiinae } \\
\hline 1 Dismorphia amphione astynome (Dalman, 1823) & 0 & 0 & 0 & 0 & 0 & 0 & 0 & 0 & 0 & 0 & 0 & 0 & 0 & 1 & 0 & 0 & 1 & 0 & 2 \\
\hline 2 Dismorphia astyocha Hübner, [1831] & 0 & 1 & 0 & 1 & 0 & 0 & 0 & 0 & 1 & 0 & 0 & 0 & 0 & 0 & 0 & 0 & 0 & 1 & 4 \\
\hline 3 Dismorphia melia (Godart, [1824]) & 0 & 0 & 0 & 0 & 0 & 0 & 0 & 0 & 0 & 0 & 0 & 0 & 0 & 0 & 0 & 1 & 0 & 0 & 1 \\
\hline 4 Dismorphia thermesia thermesia (Godart, 1819) & 0 & 0 & 3 & 4 & 3 & 0 & 0 & 0 & 0 & 0 & 2 & 0 & 0 & 0 & 0 & 0 & 0 & 0 & 12 \\
\hline 5 Enantia clarissa (Weymer, 1895) & 0 & 0 & 0 & 1 & 0 & 0 & 0 & 0 & 0 & 2 & 0 & 0 & 2 & 1 & 0 & 0 & 0 & 0 & 5 \\
\hline 6 Enantia lina psamanthe (Fabricius, 1793) & 0 & 0 & 0 & 0 & 0 & 0 & 0 & 0 & 0 & 0 & 0 & 1 & 1 & 0 & 0 & 0 & 0 & 0 & 2 \\
\hline 7 Pseudopieris nehemia nehemia (Boisduval, 1836) & 1 & 0 & 0 & 2 & 1 & 5 & 1 & 1 & 4 & 0 & 1 & 2 & 1 & 1 & 10 & 8 & 7 & 4 & 49 \\
\hline \multicolumn{20}{|l|}{ Coliadinae } \\
\hline 8 Colias lesbia lesbia (Fabricius, 1775) & 0 & 0 & 0 & 0 & 0 & 0 & 0 & 0 & 0 & 0 & 0 & 0 & 0 & 0 & 2 & 0 & 0 & 0 & 2 \\
\hline 9 Eurema albula albula (Cramer, [1776]) & 2 & 0 & 0 & 1 & 0 & 0 & 0 & 0 & 0 & 0 & 0 & 0 & 1 & 1 & 0 & 0 & 0 & 0 & 5 \\
\hline 10 Eurema arbela arbela Geyer, 1832 & 0 & 0 & 0 & 0 & 1 & 4 & 0 & 0 & 1 & 0 & 0 & 3 & 0 & 0 & 1 & 1 & 0 & 0 & 11 \\
\hline 11 Eurema deva deva (Doubleday, 1847) & 0 & 0 & 0 & 0 & 0 & 1 & 2 & 0 & 0 & 0 & 0 & 0 & 0 & 0 & 1 & 1 & 1 & 0 & 6 \\
\hline 12 Eurema elathea flavescens (Chavannes, 1850) & 0 & 0 & 0 & 0 & 0 & 3 & 0 & 0 & 0 & 0 & 2 & 0 & 0 & 0 & 0 & 0 & 0 & 0 & 5 \\
\hline 13 Phoebis argante argante (Fabricius, 1775) & 0 & 0 & 0 & 0 & 0 & 0 & 0 & 0 & 0 & 0 & 0 & 1 & 0 & 0 & 0 & 0 & 0 & 0 & 1 \\
\hline 14 Phoebis neocypris neocypris (Hübner, [1823]) & 10 & 1 & 0 & 0 & 3 & 1 & 4 & 4 & 1 & 3 & 3 & 22 & 6 & 10 & 10 & 1 & 7 & 1 & 87 \\
\hline 15 Phoebis philea philea (Linnaeus, 1763) & 0 & 0 & 0 & 0 & 0 & 1 & 0 & 0 & 0 & 0 & 0 & 4 & 1 & 0 & 1 & 0 & 1 & 0 & 8 \\
\hline
\end{tabular}


FAMILY/Subfamily/Tribe/Specie

16 Phoebis sennae marcellina (Cramer, [1779])

17 Phoebis trite banksi (Breyer, 1939)

18 Pyrisitia leuce leuce (Boisduval, 1836)

19 Pyrisitia nise tenella (Boisduval, 1836)

\section{Pierinae}

Anthocharidini

20 *Hesperocharis erota (Lucas, 1852)

21 Hesperocharis paranensis paranensis Schaus, 1898 Pierini

22 Leptophobia aripa balidia (Boisduval, 1836)

23 Pereute antodyca (Boisduval, 1836)

24 Pereute swainsonii (Gray, 1832)

25 Tatochila autodice autodice (Hübner, 1818)

26 Theochila maenacte maenacte (Boisduval, 1836)

\section{RIODINIDAE}

\section{Nemeobiinae}

Euselasiini

1 Euselasia eucerus (Hewitson, 1872)

2 Euselasia hygenius occulta Stichel, 1919

3 *Euselasia zara (Westwood, 1851)

\section{Riodininae}

Emesidini

4 Emesis fatimella fatimella Westwood, 1851

5 Emesis mandana mandana (Cramer, [1780])

6 Emesis ocypore zelotes Hewitson, 1872

7 Emesis russula Stichel, 1910

8 Emesis satema (Schaus, 1902)

$$
\text { Eurybiini }
$$

9 lonotos alector (Geyer, 1837)

10 Ithomiola orpheus (Westwood, 1851)

11 Mesosemia odice (Godart, [1824])

12 Mesosemia rhodia (Godart, [1824])

Nymphidiini

13 Adelotypa bolena (Butler, 1867)

14 Catocyclotis sejuncta (Stichel, 1910)

15 Mycastor leucarpis (Stichel, 1925)

16 Synargis paulistina (Stichel, 1910)

17 Theope thestias Hewitson, 1860

Riodinini

18 Barbicornis basilis mona Westwood, 1851

19 Calephelis braziliensis McAlpine, 1971

20 Chalodeta theodora (C. \& R. Felder, 1862)

21 Chamaelimnas briola doryphora Stichel, 1910

22 Charis cadytis Hewitson, 1866

$23 *$ Chorinea licursis (Fabricius, 1775)

24 Lasaia agesilas agesilas (Latreille, [1809])

25 Lasaia incoides (Schaus, 1902)

26 Melanis smithiae smithiae (Westwood, 1851)

27 Melanis xenia xenia (Hewitson, 1853)

28 Parcella amarynthina (C. \& R. Felder, [1865])

29 Rhetus periander eleusinus Stichel, 1910

30 Riodina lycisca (Hewitson, 1853)

31 Syrmatia nyx (Hübner, [1817])

Symmachiini

32 Mesene pyrippe sanguilenta Stichel, 1910

33 Mesenesp. $n$.

34 Stichelia bocchoris suavis (Stichel, 1911)

35 Symmachia arion (C. \& R. Felder, 1865) $\begin{array}{lllllllllllllllllll}\text { A1 } & \text { A2 } & \text { A3 } & \text { A4 } & \text { A5 } & \text { A6 } & \text { A7 } & \text { A8 } & \text { A9 } & \text { B1 } & \text { B2 } & \text { B3 } & \text { B4 } & \text { B5 } & \text { C1 } & \text { C2 } & \text { C3 } & \text { C4 } & \text { Total }\end{array}$

$\begin{array}{ccccccccccccccccccc}0 & 0 & 0 & 0 & 1 & 1 & 1 & 0 & 0 & 0 & 0 & 0 & 0 & 0 & 1 & 0 & 0 & 0 & 4 \\ 0 & 0 & 0 & 0 & 0 & 0 & 0 & 0 & 0 & 0 & 1 & 0 & 0 & 0 & 0 & 0 & 0 & 0 & 1 \\ 0 & 0 & 0 & 0 & 0 & 0 & 1 & 1 & 1 & 0 & 0 & 0 & 0 & 0 & 8 & 4 & 1 & 0 & 16 \\ 1 & 0 & 0 & 0 & 0 & 0 & 0 & 1 & 0 & 0 & 0 & 0 & 0 & 0 & 1 & 0 & 1 & 0 & 4\end{array}$

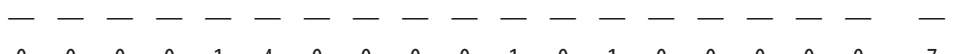

$\begin{array}{llllllllllllllllllll}0 & 0 & 0 & 0 & 0 & 1 & 2 & 1 & 0 & 2 & 7 & 2 & 4 & 3 & 0 & 0 & 2 & 0 & 24\end{array}$

$\begin{array}{lllllllllllllllllll}0 & 0 & 0 & 0 & 0 & 0 & 0 & 0 & 0 & 0 & 0 & 0 & 0 & 0 & 1 & 0 & 0 & 0 & 1\end{array}$

$\begin{array}{lllllllllllllllllll}0 & 0 & 0 & 2 & 0 & 0 & 0 & 2 & 0 & 0 & 0 & 0 & 0 & 0 & 0 & 0 & 0 & 0 & 4\end{array}$

$\begin{array}{lllllllllllllllllll}0 & 0 & 0 & 0 & 0 & 0 & 0 & 0 & 0 & 0 & 0 & 0 & 2 & 0 & 1 & 0 & 0 & 0 & 3\end{array}$

$\begin{array}{lllllllllllllllllll}0 & 0 & 0 & 0 & 1 & 1 & 1 & 1 & 0 & 0 & 3 & 0 & 0 & 6 & 7 & 1 & 1 & 0 & 22\end{array}$

\section{6}




\section{APPENDIX III}

Butterflies richness and abundance in the studied site $F=$ Fragments $(A, B, C)$

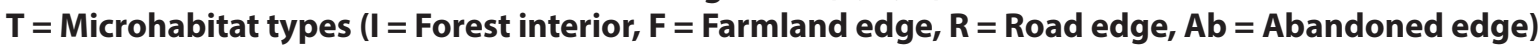

\begin{tabular}{|c|c|c|c|c|c|}
\hline $\mathbf{F}$ & Abundance & Richness & Estimated richness (95\%) & Singletons & Doubletons \\
\hline A & 3846 & 363 & 303.676 & 79 & 50 \\
\hline B & 2125 & 284 & 261.73 & 63 & 52 \\
\hline C & 1969 & 284 & 273.867 & 86 & 39 \\
\hline$T$ & Abundance & Richness & Estimated richness (95\%) & Singletons & Doubletons \\
\hline I & 2062 & 227 & 201.673 & 61 & 34 \\
\hline $\mathrm{F}$ & 1764 & 269 & 264.176 & 84 & 34 \\
\hline $\mathrm{R}$ & 1941 & 299 & 289.163 & 82 & 50 \\
\hline$A b$ & 2173 & 338 & 327.172 & 91 & 48 \\
\hline Site sampled & Abundance & Richness & Estimated richness (90\%) & Singletons & Doubletons \\
\hline$A 1 / I$ & 390 & 120 & 142.325 & 56 & 21 \\
\hline $\mathrm{A} 2 / \mathrm{l}$ & 326 & 83 & 86.648 & 36 & 19 \\
\hline$A 3 / 1$ & 389 & 93 & 99.432 & 43 & 17 \\
\hline $\mathrm{A} 4 / \mathrm{I}$ & 299 & 84 & 100.889 & 42 & 15 \\
\hline$A 5 / F$ & 373 & 114 & 125.102 & 49 & 28 \\
\hline$A 6 / R$ & 490 & 190 & 243.988 & 94 & 40 \\
\hline$A 7 / R$ & 499 & 140 & 148.573 & 59 & 32 \\
\hline $\mathrm{A} 8 / \mathrm{Ab}$ & 500 & 168 & 190.231 & 74 & 42 \\
\hline $\mathrm{A} 9 / \mathrm{Ab}$ & 580 & 180 & 208.411 & 80 & 33 \\
\hline $\mathrm{B} 1 / \mathrm{I}$ & 312 & 113 & 157.015 & 61 & 21 \\
\hline $\mathrm{B} 2 / \mathrm{F}$ & 459 & 132 & 148.106 & 58 & 24 \\
\hline $\mathrm{B} 3 / \mathrm{R}$ & 553 & 143 & 149.036 & 59 & 23 \\
\hline $\mathrm{B} 4 / \mathrm{R}$ & 399 & 154 & 182.644 & 72 & 40 \\
\hline $\mathrm{B} 5 / \mathrm{Ab}$ & 402 & 148 & 197.868 & 75 & 26 \\
\hline $\mathrm{C} 1 / \mathrm{Ab}$ & 691 & 195 & 202.487 & 75 & 40 \\
\hline $\mathrm{C} 2 / \mathrm{F}$ & 498 & 133 & 135.681 & 51 & 28 \\
\hline $\mathrm{C} / \mathrm{F}$ & 434 & 150 & 194.529 & 72 & 23 \\
\hline C4/I & 346 & 107 & 146.982 & 57 & 16 \\
\hline
\end{tabular}


APPENDIX IV

\section{Butterflies species indicators in the studied area (only species with $\mathbf{n} \geq \mathbf{1 0}$ were included) \\ H: Habitat = E: edge, l: forest interior \\ Habits and Host plants information were obtained from literature cited below this table}

\begin{tabular}{|c|c|c|c|c|c|}
\hline Family/Subfamily/Specie & H & $\mathbf{P}$ & & Habits & Host plants \\
\hline \multicolumn{6}{|l|}{ Hesperiidae/Eudaminae } \\
\hline Spicauda teleus & $E$ & 0.001 & $* * *$ & Open areas ${ }^{1}$ & $\begin{array}{l}\text { Cyperaceae; Poaceae: Coelorachis sp., Cynodon dactylon, Oryza latifolia, Panicum maximum, } \\
\text { Sorghum halepense; Fabaceae: Schrankia sp., Glycine max, Phaseolus vulgaris, Pisum sativum }{ }^{5} \text {. }\end{array}$ \\
\hline \multicolumn{6}{|l|}{ Hesperiinae } \\
\hline Anthoptus epictetus & $\mathrm{E}$ & 0.016 & * & Disturbed forest $^{3}, 0$ pen areas ${ }^{1}$ & Poaceae $e^{5}$ \\
\hline Callimormus rivera & $\mathrm{E}$ & 0.002 & $* *$ & & \\
\hline Cobalopsis miaba & I & 0.001 & $* * *$ & & \\
\hline Conga iheringii & $\mathrm{E}$ & 0.014 & * & Clearings $^{1}$ & \\
\hline Corticea lysias potex & $\mathrm{E}$ & 0.006 & ** & Open areas $^{3}$ & \\
\hline Corticea mendica ssp. n. & $\mathrm{E}$ & 0.039 & * & & \\
\hline Corticea oblinita & $\mathrm{E}$ & 0.004 & $* *$ & & \\
\hline Cymaenes tripunctata tripunctata & $\mathrm{E}$ & 0.001 & $* * *$ & Open areas ${ }^{1,3}$ & \\
\hline Lucida lucia lucia & I & 0.015 & * & & \\
\hline Polites vibex catilina & $\mathrm{E}$ & 0.018 & * & Open areas ${ }^{1,3}$ & Poaceae $^{3}$; Smilacaceae: Smilax spp.; Solanaceae: Solanum variabile ${ }^{5}$ \\
\hline Pompeius pompeius & $\mathrm{E}$ & 0.011 & * & Open areas ${ }^{1,3}$ & Poaceae ${ }^{5}$ \\
\hline Synapte silius & I & 0.017 & * & Forest $^{3}$, Clearings $^{1}$ & Arecaceae: Syagrus romanzoffiana ${ }^{5}$ \\
\hline Thargella evansi & I & 0.014 & * & Forest $^{3}$ & \\
\hline Thespieus jora & $\mathrm{E}$ & 0.003 & $* *$ & Clearings $^{1}$ & \\
\hline Vehilius inca & $\mathrm{E}$ & 0.03 & * & Disturbed areas $^{3}, 0$ pen areas ${ }^{1}$ & Poaceae: Panicum maximum, Rottboellia cochinchinensis ${ }^{5}$ \\
\hline Vehilius stictomenes stictomenes & $\mathrm{E}$ & 0.002 & $* *$ & Open areas $^{3}$ & Poaceae: Paspalum spp. ${ }^{5}$ \\
\hline Vinius letis & I & 0.002 & $* *$ & Open areas ${ }^{1}$ & \\
\hline Wallengrenia premnas & $\mathrm{E}$ & 0.037 & * & Open areas ${ }^{1}$ & Poaceae: Echinochloa crus-galli, Leersia hexandra, Oryza sativa, Stenotaphrum secundatum ${ }^{5}$ \\
\hline \multicolumn{6}{|l|}{ Pyrginae } \\
\hline Achlyodes mithridates thraso & $\mathrm{E}$ & 0.001 & $* * *$ & & Rutaceae: Citrus spp., Zanthoxylum spp. ${ }^{5}$ \\
\hline Burnsius orcus & $\mathrm{E}$ & 0.001 & $* * *$ & Open areas $^{1}$ & $\begin{array}{l}\text { Malvaceae: Abelmoschus esculentus, Alcea rosea, Althaea sp., Hibiscus sp., Malva spp., } \\
\text { Malvastrum sp., Sida spp. }{ }^{5}\end{array}$ \\
\hline Helias phalaenoides palpalis & $\mathrm{E}$ & 0.038 & * & Disturbed forest ${ }^{3}$ & Verbenaceae: Citharexylum montevidense ${ }^{5}$ \\
\hline Heliopetes alana & $\mathrm{E}$ & 0.011 & * & Open areas ${ }^{1,3}$ & Malvaceae: Sida sp. ${ }^{3}$ \\
\hline Heliopetes omrina & $\mathrm{E}$ & 0.01 & ** & Open areas ${ }^{1,3}$ & $\begin{array}{l}\text { Convolvulaceae: Convolvulus arvensis, Ipomoea spp.; Malvaceae: Abutilon spp., Pavonia } \\
\text { spinifex, Sida sp. }{ }^{5}\end{array}$ \\
\hline Staphylus musculus & $\mathrm{E}$ & 0.023 & * & Clearings $^{1}$ & Amaranthaceae: Gomphrena spp. ${ }^{5}$ \\
\hline Trina geometrina geometrina & $\mathrm{E}$ & 0.001 & $* * *$ & Disturbed forest ${ }^{3}$ & Malvaceae: Sida rhombifolia ${ }^{5}$ \\
\hline Xenophanes tryxus & $\mathrm{E}$ & 0.048 & * & Open areas $^{3}$ & Fabaceae: Glycine max; Malvaceae: Hibiscus sp., Malachra spp., Pavonia spp. ${ }^{5}$ \\
\hline \multicolumn{6}{|l|}{ Tagiadinae } \\
\hline Celaenorrhinus eligius punctiger & I & 0.003 & ** & Humid forest $t^{3}$ & Acanthaceae: Justicia carnea ${ }^{11}$ \\
\hline \multicolumn{6}{|l|}{ Lycaenidae/Theclinae } \\
\hline Arawacus meliboeus & $\mathrm{E}$ & 0.002 & $* *$ & & Solanaceae: Solanum spp.,11 \\
\hline Calycopis caulonia & $\mathrm{E}$ & 0.022 & * & & Cannabaceae: Celtis iguanaea ${ }^{5}$, leaf detritus ${ }^{4}$ \\
\hline Cyanophrys remus & $\mathrm{E}$ & 0.016 & * & & Fabaceae: Calliandra parvifolia ${ }^{5}$ \\
\hline Strephonota elika & I & 0.016 & * & & \\
\hline \multicolumn{6}{|l|}{ Nymphalidae/Apaturinae } \\
\hline Doxocopa laurentia laurentia & $\mathrm{E}$ & 0.031 & * & & Cannabaceae: Celtis sp., ${ }^{5,11}$ \\
\hline \multicolumn{6}{|l|}{ Biblidinae } \\
\hline Biblis hyperia nectanabis & $\mathrm{E}$ & 0.036 & * & & Euphorbiaceae: Tragia spp. ${ }^{5}$ \\
\hline Eunica eburnea & $\mathrm{E}$ & 0.026 & * & & Euphorbiaceae: Sebastiania commersoniana ${ }^{5}$ \\
\hline \multicolumn{6}{|l|}{ Danainae } \\
\hline Episcada carcinia & I & 0.004 & $* *$ & Dense forest ${ }^{3}$ & Solanaceae: Solanum spp. ${ }^{3}$ \\
\hline Episcada hymenaea hymenaea & I & 0.025 & * & & Solanaceae: Cestrum spp., Solanum spp. ${ }^{5}$ \\
\hline Epityches eupompe & I & 0.018 & * & Fragments $^{6}$ & $\begin{array}{l}\text { Solanaceae: Acnistus arborescens, Athenaea picta, Aureliana lucida, Brunfelsia australis, } \\
\text { Cestrum spp., Physalis neesiana, Solanum spp., Vassobia breviflora, Witheringia }{ }^{5}\end{array}$ \\
\hline Hypoleria adasa adasa & I & 0.001 & $* * *$ & Fragments $^{6}$ & Solanaceae: Cestrum spp. ${ }^{5}$ \\
\hline Hypothyris euclea laphria & I & 0.013 & * & & Solanaceae: Solanum spp. ${ }^{5}$ \\
\hline Pseudoscada erruca & l & 0.003 & $* *$ & Fragments $^{6}$ & Solanaceae: Brunfelsia spp., Cestrum spp., Sessea spp. ${ }^{5}$ \\
\hline
\end{tabular}




\begin{tabular}{|c|c|c|c|c|c|}
\hline Family/Subfamily/Specie & H & $\mathbf{P}$ & & Habits & Host plants \\
\hline Pteronymia sylvo & 1 & 0.001 & $* * *$ & & Solanaceae: Brunfelsia australis, Cestrum spp., Solanum spp. ${ }^{5}$ \\
\hline \multicolumn{6}{|l|}{ Heliconiinae } \\
\hline Actinote carycina & $\mathrm{E}$ & 0.001 & $* * *$ & Disturbed areas ${ }^{3}$ & $\begin{array}{l}\text { Asteraceae: Eupatorium spp., Mikania micranta, Symphyopappus reticulatus, Trichogonia } \\
\text { gardneri }{ }^{5}\end{array}$ \\
\hline Actinote melanisans & $\mathrm{E}$ & 0.007 & $* *$ & Disturbed areas $^{4}$ & Asteraceae: Mikania spp. ${ }^{5}$ \\
\hline Dione moneta moneta & $\mathrm{E}$ & 0.046 & * & & Passifloraceae: Passiflora spp. ${ }^{5}$ \\
\hline \multicolumn{6}{|l|}{ Limenitidinae } \\
\hline Adelpha syma & $\mathrm{E}$ & 0.047 & * & Disturbed areas $^{3}$ & Rosaceae: Rubus spp.; Rubiaceae: Cephalanthus glabratus ${ }^{5}$ \\
\hline \multicolumn{6}{|l|}{ Libytheinae } \\
\hline Libytheana carinenta & $\mathrm{E}$ & 0.03 & * & & Cannabaceae: Celtis spp. ${ }^{2,5}$ \\
\hline \multicolumn{6}{|l|}{ Nymphalinae } \\
\hline Anartia amathea roeselia & $\mathrm{E}$ & 0.03 & * & Disturbed areas $^{3}$ & Acanthaceae: Acanthus sp., Dicliptera spp., Justicia spp., Ruellia spp. ${ }^{5}$ \\
\hline Chlosyne lacinia saundersi & $\mathrm{E}$ & 0.036 & * & Open areas ${ }^{3}$ & $\begin{array}{l}\text { Amaranthaceae: Amaranthus hybridus; Asteraceae: Acanthospermum spp., Ambrosia spp., } \\
\text { Bidens pilosa, Emilia sonchifolia, Eupatorium sp., Galinsoga parviflora, Helianthus spp., } \\
\text { Parthenium hysterophorus, Senecio brasiliensis, Sonchus oleraceus, Sphagneticola trilobata, } \\
\text { Synedrella nodiflora, Verbesina spp., Vernonia sp., Viguiera sp., Wedelia glauca, Xanthium } \\
\text { strumarium; Fabaceae: Glycine max; Rubiaceae: Richardia brasiliensis }{ }^{5}\end{array}$ \\
\hline Hypanartia bella & $\mathrm{E}$ & 0.01 & $* *$ & Mountain forest ${ }^{3}$ & $\begin{array}{l}\text { Cannabaceae: Celtis spp., Trema micranta; Urticaceae: Boehmeria spp., Parietaria debilis, } \\
\text { Phenax laevigatus, Urtica spathulata }{ }^{5}\end{array}$ \\
\hline Hypanartia lethe & $\mathrm{E}$ & 0.015 & $*$ & Clearings $^{3}$ & $\begin{array}{l}\text { Cannabaceae: Celtis spp., Trema micranta; Urticaceae: Boehmeria spp., Phenax sp., Urera } \\
\text { baccifera }^{5}\end{array}$ \\
\hline Ortilia ithra & $\mathrm{E}$ & 0.001 & $* * *$ & Disturbed forest ${ }^{3}$ & $\begin{array}{l}\text { Acanthaceae: Acanthus spp., Asystasia gangetica, Dicliptera sericea, Fittonia spp., Justicia spp., } \\
\text { Ruellia spp. }^{5}\end{array}$ \\
\hline Ortilia orthia & $\mathrm{E}$ & 0.004 & $* *$ & Disturbed forest ${ }^{3}$ & $\begin{array}{l}\text { Acanthaceae: Ruellia coerulea; Asteraceae: Aster spp., Calistephus chinensis, Noticastrum } \\
\text { diffusum }^{5}\end{array}$ \\
\hline Tegosa claudina & $E$ & 0.002 & $* *$ & Disturbed areas ${ }^{3}$ & $\begin{array}{l}\text { Acanthaceae: Ruellia sp.; Asteraceae: Mikania spp.; Scrophulariaceae: Verbascum spp.; } \\
\text { Verbenaceae: Glandularia spp., Verbena spp. }{ }^{5}\end{array}$ \\
\hline Vanessa braziliensis & $\mathrm{E}$ & 0.004 & $* *$ & Open areas $^{3}$ & $\begin{array}{l}\text { Asteraceae: Achyrocline spp., Antennaria spp., Gamochaeta spp., Gnaphalium spp., } \\
\text { Pseudognaphalium obtusifolium }{ }^{5}\end{array}$ \\
\hline \multicolumn{6}{|l|}{ Satyrinae } \\
\hline Blepolenis batea & $E$ & 0.035 & * & Matrix specialist $^{9}$ & Cyperaceae; Poaceae; Arecaceae: Butia capitata, Syagrus romanzoffiana ${ }^{5}$ \\
\hline Carminda paeon & 1 & 0.004 & ** & Forest $^{3}$ & Poaceae: Bambusoideae ${ }^{5}$ \\
\hline Euptychoides castrensis & 1 & 0.017 & * & Clearings $^{3}$ & Cyperaceae $^{3}$ \\
\hline Godartiana muscosa & 1 & 0.001 & $* * *$ & Forest $^{10}$ & Cyperaceae; Poaceae: Setaria poiretian ${ }^{5,10}$ \\
\hline Paryphthimoides poltys & 1 & 0.008 & ** & Early-regrowth forest $^{8}$ & Poaceae $e^{3}$ \\
\hline Taygetis ypthima & 1 & 0.025 & * & Forest specialist $^{9}$ & Poaceae: Bambusoideae ${ }^{5}$ \\
\hline Yphthimoides ordinaria & $\mathrm{E}$ & 0.042 & * & Open areas $^{7}$ & Poaceae: Axonopus compressus, Panicum maximum ${ }^{7}$ \\
\hline \multicolumn{6}{|l|}{ Pieridae/Coliadinae } \\
\hline \multicolumn{6}{|l|}{ Dismorphiinae } \\
\hline Pseudopieris nehemia nehemia & $\mathrm{E}$ & 0.026 & * & & Fabaceae: Acacia spp., Calliandra spp., ${ }^{5}$ Senegalia sp. ${ }^{11}$ \\
\hline \multicolumn{6}{|l|}{ Pierinae } \\
\hline Theochila maenacte maenacte & $\mathrm{E}$ & 0.019 & * & & Cruciferae $^{5}$ \\
\hline \multicolumn{6}{|l|}{ Riodinidae/Riodininae } \\
\hline Barbicornis basilis mona & $\mathrm{E}$ & 0.026 & * & & Sapotaceae: Pouteria gardneriana; Cannabaceae: Celtis sp. ${ }^{5}$ \\
\hline Calephelis braziliensis & $\mathrm{E}$ & 0.016 & $*$ & & \\
\hline Emesis russula & $\mathrm{E}$ & 0.035 & * & & $\begin{array}{l}\text { Apocynaceae: Aspidosperma tomentosum; Aquifoliaceae: Ilex paraguariensis; Burceraceae: } \\
\text { Protium ovatum; Connaraceae: Rourea induta; Erythroxylaceae: Erythroxylum spp.; } \\
\text { Euphorbiaceae: Maprounea guianensis, Ricinus communis; Malpighiaceae: Byrsonima spp.; } \\
\text { Moraceae: Ficus carica; Myrtaceae: Eugenia spp.; Salicaceae: Casearia sylvestris; Sapotaceae: } \\
\text { Pouteria ramiflora }\end{array}$ \\
\hline Mesosemia rhodia & 1 & 0.004 & $* *$ & & \\
\hline
\end{tabular}

\footnotetext{
${ }^{1}$ Biezanko \& Mielke (1973)

${ }^{2}$ Biezanko et al. (1974)

${ }^{3}$ Brown (1992)

${ }^{4}$ Duarte et al. (2005)

${ }^{5}$ Becalloni et al. (2008)

${ }^{6}$ Uehara-Prado \& Freitas (2009)

${ }^{7}$ Freitas et al. (2012)

${ }^{8}$ Ribeiro et al. (2012)

${ }^{9}$ Brito et al. (2014)

${ }^{10}$ Zacca et al. (2017)

${ }^{11}$ Orlandin et al. (in prep.).

Significance level: ${ }^{* * *}, P<0.001 ;{ }^{* *}, P<0.01 ;{ }^{*}, P<0.05$
} 